\title{
KECK HIRES OBSERVATIONS OF THE QSO FIRST J104459.6+365605: EVIDENCE FOR A LARGE SCALE OUTFLOW
}

\author{
Martijn de Kool, Nahum Arav, Robert H. Becker ${ }^{2,3}$, Michael D. Gregg ${ }^{2,3}$, \\ Richard L. White, Sally A. Laurent-Muehleisen ${ }^{2,3}$, Trevor Price ${ }^{2,3}$ and Kirk T. Korista ${ }^{5}$
}

\begin{abstract}
This paper presents an analysis of a Keck HIRES spectrum of the QSO FIRST J104459.6+365605, covering the rest wavelength range from 2260 to $2900 \AA$. The line of sight towards the QSO contains two clusters of outflowing clouds that give rise to broad blue shifted absorption lines. The outflow velocities of the clouds range from -200 to $-1200 \mathrm{~km} \mathrm{~s}^{-1}$ and from -3400 to $-5200 \mathrm{~km} \mathrm{~s}^{-1}$, respectively. The width of the individual absorption lines ranges from 50 to more than $1000 \mathrm{~km} \mathrm{~s}^{-1}$.

The most prominent absorption lines are those of $\mathrm{Mg}$ II, MgI, and Fe II, and Mn II is also present. The low ionization absorption lines occur at the same velocities as the most saturated MgII lines, showing that the Fe II , Mg I and Mg II line forming regions must be closely associated. Many absorption lines from excited states of Fe II are present, allowing a determination of the population of several low lying energy levels. The populations of the excited levels are found to be considerably smaller than expected for LTE, and imply an electron density in the Fe II line forming regions of $n_{e} \sim 4 \times 10^{3}$ $\mathrm{cm}^{-3}$.

Modelling the ionization state of the absorbing gas with this value of the electron density as a constraint, we find that the distance between the Fe II and $\mathrm{Mg}$ I line forming region and the continuum source is $\sim 7 \times 10^{2}$ parsec. From the correspondence in velocity between the Fe II , MgI and Mg II lines we infer that the Mg II lines must be formed at the same distance. The Mg II absorption fulfills the criteria for Broad Absorption Lines defined by Weymann et al. (1991). Therefore the distance we find between the Mg II line forming region and the continuum source is surprising, since BALs are generally thought to be formed in outflows at a much smaller distance from the nucleus.
\end{abstract}

Subject headings: quasars: absorption lines

\footnotetext{
${ }^{1}$ RSAA, Mt. Stromlo Observatory, Cotter Road, Weston ACT 2611, Australia; dekool@mso.anu.edu.au

${ }^{2}$ IGPP,LLNL, L-413, P.O. Box 808, Livermore, CA 94550

${ }^{3}$ Physics Department, University of California, Davis, CA 95616

${ }^{4}$ Space Telescope Science Institute, Baltimore, MD 21218

${ }^{5}$ Physics Department, Western Michigan University
} 


\section{INTRODUCTION}

Many QSO rest-frame UV spectra exhibit blue shifted absorption lines that are indicative of an outflow. The properties of these absorption lines vary over a wide range, from very broad ( $>10,000$ $\mathrm{km} \mathrm{s}^{-1}$ ) absorption lines, usually observed in high ionization species like C IV and O vi to fairly narrow $\left(\sim 30 \mathrm{~km} \mathrm{~s}^{-1}\right)$ lines from the same high ionization species, or from low-ionization species like Fe II and C II. The absorption lines are often divided in two classes. The first class contains the Broad Absorption Lines (BALs)(Weymann, Turnshek \& Christiansen 1985, Turnshek 1988) which are broad absorption troughs, and can span a large range of outflow velocities up to several times $10^{4} \mathrm{~km} \mathrm{~s}^{-1}$. The second class refers to Associated Absorption Lines (AALs) (Weymann et al. 1979, Foltz et al. 1986), which have individual absorption components less than a few hundred $\mathrm{km}$ $\mathrm{s}^{-1}$ wide, and span a range of outflow velocities up to several thousand $\mathrm{km} \mathrm{s}^{-1}$. It is not obvious whether this division reflects a real bimodal distribution of absorption line properties caused by two different underlying formation mechanisms. There are many examples of QSOs that exhibit both types of lines simultaneously, or have properties that are a mixture between the two classes, eg. high velocity narrow lines or low velocity broad lines.

It is generally thought that BALs are formed relatively close to the galactic nucleus ( $\lesssim 1$ parsec), although the direct observational evidence for this is not very strong. The minimum distance separating the region in which broad absorption lines arise (the BALR) from the source of the continuum radiation is set by the observation that the Broad Emission Lines are often partly or completely absorbed by the outflow (Turnshek 1988). This places the BALR outside the Broad Emission Line Region (BELR), the size of which is now relatively well established with reverberation mapping techniques at $\sim 0.1 \mathrm{pc}$ for an average luminosity QSO. The maximum distance of the BALR from the central source is more difficult to constrain. Adopting typical, if uncertain, physical parameters for the BAL clouds it can easily be shown that unrealistically large mass and energy loss rates are implied if the lines are formed more than a few tens of parsecs from the central engine. Further support for the conjecture that the BALR is not much larger than the BELR comes from consideration of the acceleration mechanisms, which generally require that the flow starts at a distance comparable to the size of the BELR in order to reach the high speeds observed (e.g. Arav \& Li 1994). The physical conditions inside the BALR are difficult to constrain because of the extremely wide and deep absorption lines in classical BALQSOs. The width complicates the analysis because the optical depth at a certain wavelength is the convolution of the column density as a function of velocity and the multiplet structure of the line, so that the column density as a function of velocity can only be derived by a deconvolution. The large depth makes the derived column densities very sensitive to partial covering of the continuum light source.

AALs appear to be more diverse, and could arise from gas located in the AGN environment, the host galaxy, or intervening gas with a smaller cosmological redshift than the QSO. Some recent studies of AALs are Hamann, Netzer \& Shields (2000), Petitjean \& Srianand (1999), Srianand \& Petitjean (2000), Barlow \& Sargent (1997) and Ganguly et al. (1999). The analysis of AALs does not suffer as much from the problems of blending and saturation encounterd with BALs, and can give quite detailed results on the physical conditions and geometry of the absorbing gas, as demonstrated in the references above. From these studies it has become clear that the great majority of AALs show the effects of partial covering in their observed multiplet line ratios, (e.g. 
Hamann et al. 1997, Arav et al. 1999) which argues against a cosmological origin, and is most easily explained if the lines are formed in the AGN environment. However, to our knowledge no firm limits on the distance of the absorbers to the active nucleus have been derived so far.

Here we investigate the $\mathrm{z}=0.700$ QSO FIRST J104459.6+365605 (hereafter FBQS1044+3656). This object was discovered in the FIRST Bright Quasar Survey (White et al 2000) which consists of 636 radio-selected quasars distributed over $\sim 2700$ square degrees. The absorption lines in the spectrum of this QSO have several unique features, allowing us to constrain the density and ionization parameter in the outflow. The spectrum contains absorption lines of $\mathrm{Mg}$ I, $\mathrm{Mg}$ II and Fe II. Some of the Fe II lines arise from low-lying excited states. Most of the Fe II lines are not very wide nor heavily saturated, behaving roughly as expected from their relative oscillator strength, making quantitive estimates of the column density possible. The derived relative populations of several low-lying energy levels of Fe II allow us to put significant constraints on the physical conditions in the absorbing clouds.

The characteristic velocity and width of the absorption lines in FBQS1044+3656 are intermediate between BALs and AALs. The Mg II absorption is very broad, and just fulfills the strict criterion applied to the C IV line by Weymann et al. 1991 to decide whether a QSO is a BALQSO. The lower ionization lines of Fe II and Mg I are narrower, and more characteristic of AALs. However, we will argue that the excellent correspondence in velocity between the strongest Mg II components and the Mg I and Fe II lines leave little doubt that they arise from the same outflowing cloud complex.

Our analysis is similar to the study of BALQSO Q0059-2735 by Wampler, Chugai \& Petitjean 1995 (hereafter referred to as WCP). Q0059-2735 is a classical BALQSO with very deep and wide BAL troughs in both high and low ionization species. Its spectrum also contains several narrow, low ionization absorption line systems, such as Fe II, Ni II, and Mn II, at velocities of a few hundred $\mathrm{km} \mathrm{s}^{-1}$. Another recent study with some similarities to ours considers narrow absorption line systems in the BALQSO APM 08279+5255 (Srianand \& Petitjean 2000), which show absorption from excited states of $\mathrm{C}$ II and $\mathrm{Si}$ II .

\section{Data Acquisition and Reduction}

On December 26, 1998 we used the High Resolution Echelle Spectrometer (HIRES, Vogt et al. 1994) on the Keck I 10-m telescope to obtain $4 \times 1800$ second exposures of FBQS1044+3656 covering 3825 - $6280 \AA$ using a 1"'1 wide slit. The orders overlap up to $5128 \AA$, beyond which small gaps occur between orders. The slit was rotated to the parallactic angle to minimize losses due to differential atmospheric refraction. The observing conditions were excellent with sub-arcsecond seeing and near-photometric skies. The spectra were extracted using routines tailored for HIRES (Barlow 2000). The resolution of the final spectrum is 4 pixels pixels FWHM, or $8.8 \mathrm{~km} \mathrm{~s}^{-1}$ in velocity space.

The redshift of FBQS1044+3656 ( $\mathrm{z}=0.700)$ was determined by fitting a Gaussian to the Mg II broad emission line. Because the blue wing of the Mg II BEL is strongly affected by absorption, the redshift of the QSO rest frame is uncertain by several hundred $\mathrm{km} \mathrm{s}^{-1}$. Since all outflow velocities quoted in this paper are calculated from the difference in redshift between the absorbing gas and 
the QSO rest frame, they are uncertain by the same amount.

\section{Analysis}

In this section we will analyze the absorption lines in the spectrum of FBQS1044+3656, deriving the column densities of the observed species. In the next section, these column densities will be used to derive the physical condition in the outflow. An overview of the HIRES spectrum is shown in figure 1. A low resolution spectrum of FBQS1044+3656 covering a larger wavelength range has been published in White et al. 2000.

\section{1. $\quad$ Mg I lines}

The Mg I line at $\lambda 2852.96 \AA$ is a singlet transition from the ground level. The line profile as a function of velocity is shown in Figure 2, together with the profile of the Mg II absorption. The velocity scale of the Mg II absorption is based on the rest wavelength of the red component of the Mg II doublet.

We divide the Mg I absorption features into two clusters, the first ranging from -100 to -1400 $\mathrm{km} \mathrm{s}^{-1}$ (cluster 1), the second from -3500 to $-4100 \mathrm{~km} \mathrm{~s}^{-1}$ (cluster 2). Some of the components in the low-velocity cluster are not connected by any absorption, so this definition of these two clusters is purely ad hoc at this point. It will turn out however that our division appears to have a physical basis since the components of cluster 2 exhibit significant absorption from excited levels of Fe II , whereas the detection of absorption from excited levels from the components of cluster 1 is only marginal. This indicates that the physical conditions differ between the two clusters. In each cluster we have identified the two strongest components so that we can refer to these later when identifying the corresponding absorption lines of other species. Based on the assumed redshift of 0.700 for the QSO, these components correspond to an outflow speed of $-205,-1227,-3557$ and $-3860 \mathrm{~km} \mathrm{~s}^{-1}$ for components A,B,C and D respectively.

A lower limit on the column density of $\mathrm{Mg}$ I can be obtained in the optically thin approximation, valid if the line is fully resolved and the absorbing gas is covering the entire continuum source:

$$
N_{i o n} \geq \frac{m_{e} c}{\pi e^{2}} \frac{1}{\lambda_{o} f_{l u}} \int \tau(v) d v,
$$

The Mg I column density derived in this way is $1.4 \times 10^{13} \mathrm{~cm}^{-2}$ for cluster 1 and $1.0 \times 10^{13} \mathrm{~cm}^{-2}$ for cluster 2. From the analysis of the Fe II lines below, we will find that the Fe II absorbing clouds do not cover the entire continuum source. It is not clear if the same applies to the $\mathrm{Mg}$ I line, since the continuum at the position of the Fe II lines contains a large fraction of broad Fe II emission which comes from a much more extended region than the continuum from the accretion disk. Thus it is possible that the outflow covers the accretion disk source completely but the Fe II emission line regions only partially, which would lead to partial covering being observed for absorption lines in the part of the spectrum where broad Fe II line emission contributes significantly to the background source. Even if the same partial covering would apply to the Mg I line, however, this would increase 
the Mg I column density only by a factor $<2$ since the Mg I lines are significantly shallower than the saturated Fe II lines. Larger deviations could occur if the covering factor for the Mg I producing clouds is very different from that of the Fe II producing clouds. This possibility can not be excluded, but the fact that the $\mathrm{Mg}$ I line profile can be used as template for both strong and weak Fe II lines (as shown below) argues against it. The presence of Mg I in FBQS1044+3656 stands in important contrast with the absence of Mg I absorption in Q0059-2735, which was used by WCP to obtain an upper limit on the distance of the clouds to the continuum source.

\section{2. $\quad \mathrm{Mg}$ II lines}

From the comparison of Mg I and Mg II absorption as a function of velocity in Figure 2, it can be seen that the Mg II absorption extends to much higher velocities than MgI. There is a strong component centered around $-4115 \mathrm{~km} \mathrm{~s}^{-1}$ that does not appear in $\mathrm{Mg} \mathrm{I}$, and further contiguous absorption is visible up to $-5700 \mathrm{~km} \mathrm{~s}^{-1}$. At even higher velocities, some very shallow absorption systems at $-6500,-7310$ and $-7410 \mathrm{~km} \mathrm{~s}^{-1}$ are probably due to $\mathrm{Mg}$ II since both components of the doublet are visible.

The Mg II lines are heavily saturated allowing only a lower limit on the column density. Over the velocity range covered by cluster 1 and 2, we estimate lower limits on the Mg II column density of $2.610^{14} \mathrm{~cm}^{-2}$ and $5.810^{14} \mathrm{~cm}^{-2}$ respectively, but the real columns are likely to be significantly higher. The differences between the $\mathrm{Mg}$ II and $\mathrm{Mg}$ I profiles do not imply that the ionization state has to change with velocity: the column density of Mg I associated with the high velocity $\mathrm{Mg}$ II components would be too small to be detected if the Mg I/ Mg II ratio is the same as in the strongly saturated Mg II components. This interpretation is supported by the high-velocity absorption not being readily detectable in the weakest Fe II absorption lines, but being just visible in the strongest Fe II lines.

\subsection{Fe II lines}

\subsubsection{Analysis using the Mg I template}

Most of the physical constraints on the absorbing gas can be extracted from the numerous absorption lines of Fe II in the spectrum of FBQS1044+3656. Because of the blending of the Fe II

lines, most of our analysis must use a template for the column density as a function of velocity, which we derive from the unblended singlet transition of $\mathrm{Mg}$ I, an approach similar to that of Arav et al. 1999a. The template method is based on the assumption that the column density of Fe II in each energy level $k$ between velocity $v$ and $v+d v$ is proportional to the column density of $\mathrm{Mg}$ I in the same velocity interval:

$$
\frac{d N_{F e I I}^{k}}{d v}=c_{k} \frac{d N_{M g I}}{d v}
$$

The column density of $\mathrm{Mg}$ I is derived from the $\mathrm{Mg}$ I absorption line profile using

$$
\frac{d N_{M g I}}{d v}=\frac{m_{e} c}{\pi e^{2}} \frac{1}{\lambda_{M g} f_{M g}}(-\ln I(v))
$$


where $I(v)$ is the remaining flux in the normalized line profile, and $f_{M g}$ the oscillator strength of the Mg I resonance line. By using Equation 3, we implicitly assume that partial covering effects are not important for $\mathrm{Mg}$ I (see above), and that the absorption lines are fully resolved. Any quantitative analysis must make the second assumption, although it should be kept in mind that the thermal line width of $\mathrm{Fe}$ and $\mathrm{Mg}$ at the temperatures of a few thousand degrees that are expected for the line forming regions are only of order $1-2 \mathrm{~km} \mathrm{~s}^{-1}$, i.e. well below the resolution of our observations. When the constants of proportionality $c_{k}$ between the column densities of $\mathrm{Mg} \mathrm{I}$ and $\mathrm{Fe} \mathrm{II}^{k}$ are specified, we can calculate the optical depth in every Fe II line as a function of velocity, and construct a model Fe II absorption line spectrum that can be compared to the observed spectrum of FBQS1044+3656.

From a first inspection of the spectrum it is clear that the components of cluster 2 show significant absorption in excited Fe II lines, whereas the components of cluster 1 show up only very weakly in these lines. Therefore, the two clusters appear to arise from physically distinct cloud complexes. To keep the number of free parameters in our fit manageable, we will assume that the level populations do not vary between different components within each cluster, but allow for different level populations between the two clusters.

The Fe II lines considered in our study are listed in Table 1. We included all lines from the lowest energy $\left(a^{6} D\right)$ term, and the strongest lines from the next two terms $\left(a^{4} F\right.$ and $\left.a^{4} D\right)$ with lowest levels at approximately 0.2 and $1 \mathrm{eV}$, respectively. In total, we consider 43 lines arising from 10 energy levels.

The fit was obtained by first choosing values for the $c_{k}$ 's for each cluster and then comparing the predicted and observed Fe II absorption line spectra. The values of the $c_{k}$ 's were successively improved by adjusting them to reproduce the observed flux at the positions in the spectrum where the specific $c_{k}$ is best constrained. To be properly constraining, we required that the parts of the spectrum used to adjust the fit contain a cluster component that is not blended with another line, and is not very deep so that partial covering effects play as small a role as possible. Although many lines are blended, all levels give rise to some absorption components that occur in a part of the spectrum where blending is not a problem.

Note that this method of fitting a model to the observed spectrum does not try to fit individual lines, but rather level populations and thus attempts to fit all lines from a given lower level with one parameter, the column density of Fe II in the state corresponding to this lower level. Because isolated, medium depth lines arising from the level are the most accurate indicators of this column density, these are the main drivers for the fit.

The model resulting from the fit obtained in this way is compared with the observed spectrum in figure 3. The thin line represents the reconstructed spectrum based on the model parameters in Table 2. We express the model parameters in terms of relative level populations and a total column density. $\zeta_{k}$ is the level population per unit statistical weight of level $k$ relative to that of the ground level,

$$
\zeta_{k} \equiv \frac{N_{F e I I}^{k} / g_{k}}{N_{F e ~ I I}^{0} / g_{0}}
$$

$\zeta_{k}$ is equal to 1 if the levels are in LTE with $\mathrm{kT}>>\mathrm{E}_{\mathrm{k}}$. The total Fe II column density is 
$\log N_{F e ~ I I}=14.7(0.2)$ for cluster 1 and $\log N_{F e ~ I I}=15.3(0.1)$ for cluster 2 . The upper limits and error estimates were obtained by visually comparing model spectra with the observations and estimating how much deviation from either 0 or the measured value could be tolerated. Systematic effects such as errors in oscillator strength or continuum level are thus not included.

The heavily blended regions of the spectrum are well reproduced using the $c_{k}$ 's obtained from the isolated lines, proving the consistency of the result. The figure shows that for most of the absorption components the fit is satisfactory, considering that the problem is heavily overconstrained and that the number of lines that have to be reproduced is several times larger than the number of energy levels included in the fit. The most significant differences between the observed spectrum and the model that can not be removed by changing the model parameters occur in the strongest absorption lines from the ground level. These strong lines show absorption at high velocity, extending beyond the highest velocity seen in cluster 2 of $\mathrm{Mg}$ I. This extra absorption is also seen as the very deep component at $-4115 \mathrm{~km} \mathrm{~s}^{-1}$ in $\mathrm{Mg}$ II. The other major deviation between model and observation is that the strongest Fe II lines are much deeper in the model. It is impossible to obtain a good fit to the five lines originating from the ground state simultaneously. The most likely explanation for this discrepancy is partial covering of the continuum source, which fills in the bottom of the strongest lines. Note that the underprediction of flux in the deepest lines is the result of our choice to optimize the fit for medium depth lines (which are the weakest lines when considering lines from the ground level).

There are several other sources of uncertainty that may also contribute to discrepancies between the model and the observed spectrum. One is that there are still some uncertainties in the oscillator strengths of the UV Fe II absorption lines studied here. Table 1 shows a comparison of these oscillator strengths from different literature sources. In this work we have used the oscillator strengths of Fuhr, Martin \& Wiese 1988 (NIST database), which are generally in reasonable agreement with recent experimental values of Bergeson et al. 1996. The only data source that has significant outliers are the theoretical values from the Iron Project (Nahar 1995), with some discrepancies as large as a factor of 5 . For one of the important transitions for this study $(\lambda 2374.46$ $\AA$, a line from the Fe II ground level) the Nahar value is almost 2 times larger than the others. Using observations of Fe II absorption lines from the interstellar medium Cardelli \& Savage 1995 derived an oscillator strength of $0.0326(14)$ for this transition, in good agreement with the laboratory experiments of Bergeson et al. 1996. Thus the theoretical value of 0.0527 derived by Nahar 1995 is unlikely to be correct. For this transition only, we have not used the oscillator strength of Fuhr, Martin \& Wiese 1988 but instead a compromise between the two recent experimental values of 0.032. More recent theoretical calculations (Raassen \& Uylings 1998) appear to reproduce the experimental values quite well, but are not as extensive as the Iron Project results.

Another source of uncertainty is the exact level of the continuum. The continuum contains several undulations, that are likely to be due to the presence of broad Fe II emission, as can be seen from figure 1, or even more clearly from the low resolution spectrum of FBQS1044+3656 published in White et al. 2000 which covers a larger wavelength range. The relative contribution of the Fe II BELs to the continuum can be estimated from the broad trough in the spectrum around $2650 \AA$ which is actually a gap between Fe II emission line complexes (see e.g., Verner et al. 1999). This trough is also present in the LBQS composite spectrum (Francis et al. 1991), but is especially 
deep in FBQS1044+3656, indicating that at least 30\% of the apparent continuum is actually broad Fe II emission. The strongest Fe II emission lines are expected at the same wavelengths where the absorption lines occur (Verner et al. 1999), and since the main blended absorption complexes themselves are also quite broad, it might well be possible that there is emission at the position of the absorption complexes that has not been accounted for in our continuum normalization. The spectrum of J121442+280329 published in White et al. 2000 is a clear example of a QSO spectrum in which a complex interplay between Fe II absorption and emission is shaping the spectrum, and modelling of the absorption would be impossible without considering the emission lines. In the case of J121442+280329 the problem is more obvious because the emission lines are narrower. Note that the lines from the Fe II ground level are distributed over a wavelength range several $100 \AA$ wide, i.e. they are separated by much more than the width of a single Fe II broad emission line. This means each absorption line will be affected by a different set of Fe II emission lines, unlike the case of most doublet lines where the separation is much smaller than the emission line width, and the presence of broad emission is unlikely to have a strong differential effect on the continuum level between the positions of the two doublet components.

\subsubsection{Partial covering}

The five strong lines arising from the Fe II ground level can not be fitted simultaneously using the preceding template approach when it is assumed that the continuum source is completely covered by the outflow. Partial covering has been shown to be important in several other QSOs with absorption lines (e.g., Hamann, Barlow, \& Junkkarinen 1997, Barlow \& Sargent 1997, Arav et al. 1999b), usually by considering the relative depth of the two lines of one of the major doublets, such as CIV and SiIV. When relatively narrow absorption components are present so that the two lines of the doublet are visible (which implies that this type of analysis can generally only be applied to AALs and not BALs), it is possible to derive a model for the outflow in which at each velocity a fraction $C(v)$ of the continuum source is covered with a homogeneous layer of absorbing gas with optical depth $\tau_{1}(v)$ in line 1 of the doublet. This is done by solving the set of coupled equations

$$
\begin{aligned}
& I_{1}(v)=(1-C(v))+C(v) e^{-\tau_{1}(v)} \\
& I_{2}(v)=(1-C(v))+C(v) e^{-\frac{(f \lambda)_{2}}{(f \lambda)_{1}} \tau_{1}(v)},
\end{aligned}
$$

for every velocity $v$. Here $I$ is the residual intensity in the normalized spectrum, the subscripts 1 and 2 refer to the two lines of the doublet, and $(f \lambda)_{2} /(f \lambda)_{1}$ is the known ratio of the optical depths in two lines that originate from the same lower level.

The same analysis can be applied to every pair chosen from the 5 lines arising from the Fe II ground level, and if the partial covering model used is correct, then each pair should yield the same covering factor as a function of velocity, providing a strong confirmation of the simple partial covering model. Several complications crop up when analysing the Fe II lines in this way, the most serious being line blending. If one of the two lines used to solve for optical depth and covering factor is contaminated by another absorption line, the solution will be erroneous and often no solution is possible at all. A second problem is that the oscillator strengths of the lines are not accurately 
known, in contrast to the case of the doublet lines where the ratio is exactly 2 . When the optical depth is very low, small continuum errors also cause problems.

In spite of these difficulties, we can still derive some insights from such a covering factor analysis. We first consider the $C(v)$ that can be derived from the two Fe II lines at $2600 \AA$ and $2586 \AA$; these suffer the least from blending with other lines and therefore give the cleanest solutions. In figure 4, we show what fraction of the continuum source must be uncovered to make the profiles of these two lines consistent, both for cluster 1 and cluster 2. At some velocities, contamination by other lines or noise do not allow a solution for $C(v)$, and we have indicated these points with asterisks above the spectrum. To reduce the effect of noise, the spectrum was smoothed with a 5 -point moving average before solving equation 6 . The shape of the strongest line is almost entirely determined by the covering factor, as is expected on the basis of our previous result that the template fitting procedure predicts a much smaller flux at the bottom of the strongest Fe II lines than is actually observed. This applies both in cluster 1 and cluster 2 . Thus, the residual flux at the bottom of the strong lines is either due to a small part the continuum source not being completely covered by the outflow, or due to scattering of continuum light around the absorbers. In the case of FBQS1044+3656 we consider the first possibility more likely, because the strong variations of covering factor as a function of velocity are not expected if the remaining flux is due to scattered light.

The covering factor solutions based on other line pairs suffer more from all of the problems mentioned above. Nevertheless, it is very important to check whether the solutions obtained from different pairs yield more or less consistent covering factors. Cluster 1 is too much affected by blending to make a useful comparison. In figure 5, we compare the solutions for the covering factor in cluster 2 for the 5 most suitable line pairs. In view of all the uncertainties, the solutions are consistent. There is a systematic difference between the pairs including the line $2344.2 \AA$ and the other pairs, in the sense that they yield a smaller covering factor outside the deepest components in the profile. Such behavior could be caused by an error in the oscillator strength of the line 2344.2 $\AA$, an error in the continuum level, or by a wavelength dependent continuum source size. Since the line is close to the blue cut-off of the spectropgraph, the $\mathrm{S} / \mathrm{N}$ ratio at the position of the 2344.2 $\AA$ line is considerably worse than at the position of the other lines, which may also contribute to the discrepancy. Since our continuum consists of a combination of a smooth underlying continuum generated in a very small region and broad undulating Fe II emission lines from a much larger region, we consider a wavelength dependent continuum source geometry to be the most likely explanation.

In principle, the covering factor solution also yields the optical depth as a function of velocity, and hence the column density. The velocity integrated column densities derived in this way for each cluster are typically 2-3 times higher than those derived from the template fitting. Since the column densities derived from template fitting mainly depend on the weakest non-saturated lines, this difference can not be attributed to the effects of partial covering. Instead, the cause of the discrepancy is that the integrals of optical depth over velocity are dominated by large peaks that occur when the residual fluxes in the two lines used to derive the covering factor solution become almost equal, which can occur because of small continuum errors when the lines are weak, or because of another absorption line falling into the profile of the weaker line. This forces a solution with very high optical depth at this velocity. Since this is not physical, we consider the column 
densities derived from the template fitting more reliable.

We now verify that the results for the level populations obtained by the covering factor approach and the template fitting procedure are consistent. The foregoing analysis shows that the covering factor completely determines the profiles of the two strongest Fe II lines, so the covering factor as a function of velocity can be estimated from the remaining flux in these lines as,

$$
(1-C(v))=\frac{1}{2}\left[I_{2382}(v)+I_{2600}(v)\right]
$$

The thick line in Figure 3 again shows the model fit based on the parameters in Table 2, this time including the effect of the covering factor defined by equation 7 . All the lines are now wellreproduced by the model. Note that if multiple lines from gas at different velocities (and hence with different covering factors) contribute to the absorption at a given wavelength, the total remaining flux depends on whether the gas at different velocities covers the same region of the background source or not. For the model presented here, we have assumed that there is no correlation between the positions of the covered or uncovered regions for gas with different velocities. Numerically, this means that each line contributing to the absorption at a given wavelength reduces the flux by a factor $r$ equal to

$$
r=(1-C(v))+C(v) e^{-\tau(v)}
$$

\subsection{Other lines}

The only other feature that we can identify with certainty is Mn II absorption from cluster 2. All three lines from the Mn II ground state (2576.877, 2594.499 and 2606.462 §) are detected. The depth of the Mn II features is well fit if we use the Mg I absorption template, and assume that the column density of Mn II is $10^{-2}$ times the column density of Fe II $\left(\log N_{M n} I I=13.3\right)$, in good agreement with standard abundance ratios. In Figure 3 the positions of the Mn II absorption lines are indicated.

Absorption lines of FeI are absent from the spectrum, providing an important constraint on the ionization parameter of the absorbing gas. The strongest line from the Fe I ground level (2484.0209 $\AA$ rest wavelength) is not detected (see figure 3). We have determined an upper limit on the Fe I column density by trying to fit model Fe I spectra using the Mg I absorption template, and estimating by eye how much Fe I could be tolerated without the line being clearly detected. Because of the relatively smooth continuum in the relevant part of the spectrum, we are able to constrain the column density of Fe I in the ground state to $\log N_{F e}<13.0$. It would be useful to have some information on the Fe III abundance (WCP), but our wavelength coverage does not include any Fe III lines. 


\section{DISCUSSION}

\subsection{The electron density in the outflow}

Our most important result is the finding that absorption from excited levels of Fe II is detected, and that the level populations are smaller than expected for LTE. This shows that the electron density in the outflowing clouds in FBQS1044 is lower than that in the lower velocity clouds in spectrum of Q0059-2735 studied by WCP, which were found to be in LTE. An accurate electron density estimate based on the observed level populations is difficult to obtain, since only a small fraction of the radiative transition probabilities for the strongly forbidden transitions within and between the $a^{6} D, a^{4} F$ and $a^{4} D$ terms are known (Nussbaumer \& Storey 1980, Quinet, Le Dourneuf \& Zeippen 1996).

A very rough estimate of the electron density can be obtained by considering only the relative populations of the ground level and the lowest excited level $\left(E=384 \mathrm{~cm}^{-1}\right)$. This level can only decay radiatively to the ground level and not make other radiative decays with unknown transition probabilities. The electron density can be estimated assuming that there is equilibrium between collisional excitation into this level from the ground level and radiative de-excitation to the ground level. For this transition the radiative decay probability $A_{21}$ is 0.00213 (Quinet, Le Dourneuf \& Zeippen 1996), and collision strength $\Omega_{12}$ is 5.5 (Zhang \& Pradhan 1995). Taking into account the formal errors on the level populations in Table 2, and constraining the temperature of the absorbing gas to lie in the range between 300 and $10.000 \mathrm{~K}$, we obtain $\log n_{e}=3.55(0.25)$ for cluster 2 and $\log n_{e}=3.0(0.4)$ for cluster 1. Using the observed level populations and the collision strengths from Zhang \& Pradhan 1995, it is easily verified that for $\log n_{e}=3.55$ and a temperature of $5000 \mathrm{~K}$ the total collisional excitation rate out of the lowest excited level is 0.24 times the radiative decay rate to the ground level, so that its neglect is justified for this rough estimate.

We can also derive an estimate of the electron density by using the most recent attempt at modelling the level populations of Fe II as a function of density using a full multilevel calculation by Verner et al. 1999. In these models the unknown transition probabilities have been set to such a small value that they are effectively neglected. Based on these results we can make an estimate of the electron density by comparing the departure coefficient of the ground level (the only level among the ones relevant here for which results are presented in Verner et al. 1999) with the predicted value as a function of electron density. We define an "observed" departure coefficient for the ground level as

$$
b_{0, o b s}=\left(\frac{n_{0, o b s}}{\sum n_{i, o b s}}\right)\left(\frac{\sum g_{i} e^{\left(-E_{i} / k T\right)}}{g_{0}}\right)
$$

where the sum is over the levels $i$ given in table 2 , and the temperature was taken to be $5000 \mathrm{~K}$ for consistency with the Verner et al. model. For this temperature, the contribution of the higher energy levels to the partition function will be very small because of the Boltzmann factor, and their neglect is well justified. The observed departure coefficients are $\log b_{0, o b s}=0.33(0.08)$ for cluster 2 and $\log b_{0, o b s}=0.37(0.17)$ for cluster 1 . Using Figure 3 in Verner et al. 1999 we can convert these limits to $\log n_{e}=3.8(0.3)$ for cluster 2 and $\log n_{e}=3.5(0.5)$ for cluster 1 . These values are in reasonable agreement with the results obtained above by treating the ground and lowest excited level of Fe II as a two-level atom. 


\subsection{Ionization modelling}

We have used the photoionization code CLOUDY (Ferland 1996) to model the ionization state of the outflow to derive additional constraints on the absorbing gas. Our observational data yield the following constraints on the outflow in FBQS1044+3656: a Mg I column density $\sim 2 \times 10^{13} \mathrm{~cm}^{-2}$, a lower limit on the Mg II / Mg I ratio of $\sim 30$, an Fe II column density $\sim 3 \times 10^{15} \mathrm{~cm}^{-2}$, an upper limit on the Fe I column density of $\sim 10^{13} \mathrm{~cm}^{-2}$ and an estimate of the electron density of $\sim 4 \times 10^{3} \mathrm{~cm}^{-3}$. It turns out that the lower limit on the $\mathrm{Mg}$ II / Mg I ratio is not very useful, so we will concentrate on the MgI, Fe II, and Fe I column densities.

\subsubsection{Dust-free models}

We will first consider simple models of the absorbing clouds, investigating their structure as a function of ionization parameter, density and shape of the ionizing spectrum. For the moment we ignore the possible effects of dust, which can introduce significant complications. The clouds are modelled as thin constant density slabs. In order to satisfy the observational constraints, the slabs are truncated when a value of the Fe II column density of $\log N_{F e ~ I I}=15.5$ is reached. Furthermore, to satisfy the electron density constraint we have adjusted the density of the slab in each model so that the Fe II weighted mean electron density is $4 \times 10^{3} \mathrm{~cm}^{-3}$. In this way the ionization parameter, the abundances and the shape of the ionizing spectrum remain as parameters that can be adjusted to satisfy the constraints on the $\mathrm{Mg}$ I and Fe I column densities. The parameters and properties of the ionization models presented here are given in Table 3.

Figure 6 illustrates the structure of the slab as a function of ionization parameter for gas with solar composition irradiated by a standard Matthews \& Ferland (1987) AGN spectrum. When modelling the very low ionization species considered here with the CLOUDY code, one encounters the difficulty that Mg I, Fe I and Fe II can occur in gas with a temperature below the limit at which CLOUDY results are reliable. We therefore had to stop the calculation of the cloud structure at the depth where the temperature falls below $1000 \mathrm{~K}$, if this occurs before the required Fe II column density is reached. At temperatures below $1000 \mathrm{~K}$ Fe II is still the main form of Fe. This means that when the calculation is stopped because of the temperature constraint (which occurs for low ionization parameters), the column densities found are only lower limits.

The run of temperature and electron density through the slab is similar to that found in the detailed Fe II BEL models of e.g. Wills et al. (1985) and Collin-Souffrin et al. (1986), with an almost fully ionized Strömgren layer at the illuminated side of the side, followed by a partially ionized zone in which most of the column density of Fe II resides. To understand the behaviour of the Mg I column density as a function of the model parameters it is important to realize that the ionization equilibrium of $\mathrm{Mg}$ behaves slightly counter-intuitively, in the sense that the fraction of neutral Mg drops sharply behind the hydrogen ionization front. The reason for this behaviour is that the photoionization rate of $\mathrm{Mg}$ I is hardly affected by the front because the ionization potential of $\mathrm{Mg}$ I is considerably lower than that of hydrogen, whereas the recombination rate is much reduced because of the sharp drop in electron density. Therefore, the Mg I column density of the slab resides almost entirely in the ionized Strömgren layer. 
The three models with $\log U \geq-3$ all have Mg I column densities less than $10^{13} \mathrm{~cm}^{-2}$ (see Table 3), between 3 and 10 times less than observed in FBQS1044+3656. The three models with $\log U$ equal to $-4,-5$ and -6 run into the $1000 \mathrm{~K}$ temperature limit before a high enough Fe II column density is reached, so that the column densities of Fe I and Mg I are lower limits only. The model with $\log U=-6$ is excluded, since it already has an Fe I column density higher than our upper limit when the Fe II column density is only $2.510^{14} \mathrm{~cm}^{-2}$. Thus of the models presented in Figure 6, only those with $\log U=-4$ and $\log U=-5$ may explain the observed column densities in FBQS1044+3656. Although the column densities may be consistent with such a very low ionization parameter model, they are not likely to provide a good explanation for the observed Fe II lines in detail, since most of the Fe II column density would have to reside in the region with a temperature below $1000 \mathrm{~K}$, which is difficult to reconcile with the observed population of the excited state at $1873 \mathrm{~cm}^{-1}$ because the Boltzmann factor for this level should be $<0.067$. Thus we conclude that simple solar composition ionization models are not able to reproduce the observed column densities.

Model $2(\log U=-2)$ only fails to reach the observed Mg I column density by a factor of 3. Such a relatively small discrepancy may be fixed by changing the abundances from the solar values. The simplest solution is to increase the $\mathrm{Mg}$ abundance by a factor of 3 , which brings the $\mathrm{Mg}$ I column density very close to the observed value. Increasing the Fe abundance reduces the thickness of the slab for a given Fe II column density, but because most of the Mg I resides in the Strömgren layer, this hardly affects the MgI column density. Thus with an overall increase in metallicity to 3 times solar (Model 7) the observed column densities can be reproduced.

Although the column densities can be reproduced by this high metallicity model, there are two problems that remain to be resolved. The first is that these models are rather contrived in the sense that extreme fine tuning of the cloud geometry is required to obtain the correct Fe II column density. Most Fe II resides in a thin layer at the back side of the cloud, with a thickness much smaller than the thickness of the Strömgren layer, a problem also pointed out by WCP (see below). Since the thickness of the slab is set by the Fe II column density, this problem can be remedied by reducing the Fe abundance by a very significant factor. This leads to slabs with a much thicker partially ionized zone. The larger extent of the partially ionized zone does not contribute appreciably to the Mg I column density however, so that an enhanced $\mathrm{Mg}$ abundance is still required. The second problem is that the $\mathrm{MgI}$ and Fe II reside in different zones of the slab. In FBQS1044+3656, the gas along the line of sight is broken up into many clouds at different velocities, and we would not expect to see such a good correspondence between the absorption components of Fe II and $\mathrm{Mg}$ I if these lines were not formed in the same gas.

In figure 7 we investigate the sensitivity of these results to our assumptions about the shape of the ionizing spectrum. The AGN spectrum used above has a very strong EUV bump. Some more recent observation (e.g. Laor et al. 1997) suggest that in at least some active galaxies the EUV flux is considerably smaller than predicted by the Matthews \& Ferland spectrum. To investigate this effect, we have repeated some models with a simple power law spectrum with slope -1.4 between 10 microns and $50 \mathrm{keV}$, which effectively removes the EUV bump. Figure 7 compares the models with $\log U$ equal to $-2,-4$ and -6 for the two choices of ionizing spectrum. Although the temperature and electron density in the partially ionized zone are higher in the case of the power law spectrum, the column densities are very similar, so that our conclusions from Figure 6 are not very dependent on 
the exact shape of the ionizing spectrum.

\subsubsection{Models with dust}

From the ionization models above we have found that the low ionization species we have been studying are mainly found in gas with a temperature of a few thousand Kelvin. This means it is very well possible that some form of dust will be present in the outflowing clouds. As we will demonstrate, this can change the ionization models significantly. The presence of dust will have three main effects:

1. Fe is likely to be strongly depleted. This means that the hydrogen column density required to obtain the observed Fe II column density is much larger.

2. It is possible that Fe and $\mathrm{Mg}$ are depleted by different depletion factors. Thus the column density ratios between $\mathrm{Fe}$ and $\mathrm{Mg}$ ions will be changed.

3. The dust absorbs ionizing radiation, which changes the ionization equilibrium in the slab.

The following models are based on the Orion dust model in CLOUDY, which is in turn based on the work on abundances and dust properties in the Orion Nebula H II regions (Ferland 1996, Baldwin et al. 1991, Osterbrock et al. 1992). The properties of this dust model that are important for this work are the depletions of Fe and $\mathrm{Mg}(\sim 0.1$ for both $)$ and the dust opacity, which reflects

the absence of small grains. The physical conditions in the clouds we consider are more similar to those in H II regions than those in the local ISM, for which a different depletion pattern and grain size distribution has been derived(e.g Cowie \& Songaila 1986).

The results of the ionization models including dust are illustrated in Figure 8. The most obvious effect of the presence of dust is the depletion of Fe, which leads to thicker slab models with a much thicker partially ionized zone, as discussed above. However, the most important physical difference between these models and the models without dust is that the $\mathrm{Mg}$ I fraction no longer drops as steeply behind the ionization front, so that the largest contribution to the $\mathrm{Mg}$ I column density now comes from the partially ionized zone rather than the Strömgren layer. The reason for this behaviour is that the $\mathrm{Mg}$ I ionizing flux (including trapped Ly $\alpha$ ) is absorbed by dust, so that the reduction in recombination rate due to the drop in electron density is partially compensated. Thus in the models with dust the Fe II and Mg I column density reside in the same region of the slab, whereas they are separated in the models without dust. Since the partially ionized zone now contributes to the Mg I column, the observed column can be explained with a much smaller $\mathrm{Mg}$ abundance than in the models without dust.

The largest Mg I column density for the given Fe II column density is produced for $\log U=-3$ (Model 13). Even for this model it is still necessary to assume that Mg is three times less depleted than in the standard Orion dust model (i.e. by a factor of 0.33 instead of 0.1 ) to reproduce the observed Mg I column. For lower ionization parameters the models run into the $1000 \mathrm{~K}$ temperature limit again, which means that most Fe II would be too cold to explain the Fe II excitation, as 
discussed above. Higher ionization parameters can also reproduce the Mg I column density if we allow $\mathrm{Mg}$ to be even less depleted. However, the models with larger ionization parameters are effectively excluded by another constraint, which is that the spectrum of FBQS1044+3656 does not appear to suffer from very strong extinction. The extinction at $2500 \AA$ predicted by the models with dust is given in Table 3. A comparison of the spectrum of FBQS1044+3656 with the mean QSO template of Weymann et al. 1991 shows that the extinction at $2500 \AA$ is not likely to be larger than 1.0 magnitude, unless the spectrum is intrinsically very steeply rising towards shorter wavelengths. This excludes the models with higher ionization parameters than $\log U=-3$.

All models with $\log U>-6$ have Fe I column densities that lie well below our upper limit. We conclude that models with dust can explain the observed properties of the outflow reasonably well, and do not suffer from the problems with an improbable cloud geometry and separation of the Mg I and Fe II producing zones encountered in the high metallicity models without dust.

\subsection{The size of the outflow}

For the cosmological parameters $H_{0}=50 \mathrm{~km} \mathrm{sec}^{-1} \mathrm{Mpc}^{-1}, \Omega=1$ and $\Lambda=0$, the absolute B magnitude of FBQS1044+3656 is $M_{B}=-26.2$. Assuming further that the shape of the continuum is close to the typical AGN spectrum described by Matthews \& Ferland (1987), we estimate the luminosity of FBQS1044+3656 to be close to the canonical QSO value of $10^{46} \mathrm{erg} \mathrm{s}^{-1}$. In table 3 , the distance between the source of ionizing radiation and the absorbing cloud corresponding to each model is tabulated assuming this luminosity. Both the high metallicity models without dust and the dusty models require that the outflow is situated at a distance of several hundred parsecs from the source of ionizing radiation, i.e typical of the QSO narrow line region. This distance is about two orders of magnitude larger than the distance derived by WCP for the Fe II absorbing clouds in the BALQSO Q0059-2735.

This large size of the outflow raises several questions. The velocity of the outflow is considerably larger than that of material in the narrow line region, and is more typical of the BEL clouds that are situated at only 0.1 parsec from the nucleus. The combination of a large distance and the very small cloud size implied by partial covering re-emphasizes the problems associated with the stability of small, high speed clouds with a very low filling factor, which have been discussed extensively in the context of the formation of broad absorption lines (Weymann, Turnshek \& Christiansen 1985, Begelman, de Kool \& Sikora 1991, Weymann 1997). Although the small implied sizes of the clouds are hard to understand theoretically, we do point out that the small transverse cloud size implied by partial covering is consistent with the physical thickness of $\sim 10^{16} \mathrm{~cm}$ of the slabs in our ionization models as derived from the hydrogen densities and column densities.

One of the main objections to large distances for BAL-like outflows comes from constraints on the mass and energy involved using the typical parameters of high-ionization BALs. The low ionization absorption lines studied in this paper, however, have rather low velocity and equivalent width compared to typical high-ionization BALs. We can estimate the mass loss rate and kinetic energy of the outflow for the specific case of FBQS1044+3656 to see if they are unreasonably large. 
The mass involved in the low ionization outflow can be estimated from

$$
M_{\text {outflow }} \gtrsim 10^{7} N_{21} R_{21}^{2} f_{\text {cov }} \mathrm{M}_{\odot}
$$

where $N_{21}$ is the hydrogen column density normalized to $10^{21} \mathrm{~cm}^{-2}, R_{21}$ the size of the outflow in units of $10^{21} \mathrm{~cm}$ and $f_{\text {cov }}$ is the global covering factor of the outflow. Since Fe II absorption is not very common in QSOs, $f_{\text {cov }}$ could be as small as $10^{-2}$ for Fe II absorption-producing outflows. Alternatively the outflows may be short lived phenomena, in which case the constraints on the mass loss rate are also less severe. Taking the parameters of our preferred Models 7 or 15 and estimating the flow time to be $R / v$, the implied mass outflow rate is $70 f_{\text {cov }} \mathrm{M}_{\odot} \mathrm{yr}^{-1}$ and the kinetic power of the outflow is $3 \times 10^{44} f_{\text {cov }}$ erg s $\mathrm{s}^{-1}$. These are values that do not argue strongly against the large size of the outflow.

\subsection{Is FBQS1044+3656 a BALQSO?}

As mentioned in the Introduction, the characteristics of the absorption lines in FBQS1044+3656 are intermediate between those of BALs and AALs. Weymann et al. (1991) classified a QSO as a BALQSO if the C IV line profile showed a continuous absorption of at least $10 \%$ in depth spanning more than $2000 \mathrm{~km} \mathrm{~s}^{-1}$ in velocity, discounting absorption closer than $3000 \mathrm{~km} \mathrm{~s}^{-1}$ bluewards of the emission peak. The Mg II absorption in FBQS1044+3656 meets these criteria, even when a correction is made for the fact that the doublet separation in $\mathrm{Mg}$ II is larger than in C IV . The total width of the Mg II absorption is about the same as in Q0059-2735 which exhibits extremely strong BALs in the higher ionization lines, and it is an established property of low ionization BALQSOs that the absorption troughs of higher ionization species like $\mathrm{C}$ IV or O VI are generally much wider than the Mg II absorption (Weymann et al. 1991). Thus, considering the properties of the Mg II absorption alone FBQS1044+3656 should be classified as a BALQSO. In the standard picture of BALQSOs, the absorption lines are formed in an outflow with a typical size of a few parsec.

The characteristics of the very low ionization lines of Mg I and Fe II are typical of AALs (Weymann et al. 1979, Foltz et al. 1986), for which no firm distance scale has been established so that the distance of a few hundred parsec found in this paper is perhaps not a surprise. The remarkable fact lies in the excellent correspondence in velocity between the strongest $\mathrm{Mg}$ II features and the Mg I and Fe II absorption lines, which establishes without a doubt that the very low ionization lines and the Mg II lines are formed in the same outflow. The association of the Fe II and Mg I absorption with the Mg II BAL is much clearer in FBQS1044+3656 than in Q0059-2735, which only shows a number of relatively narrow Fe II components with outflow speeds of a few hundred kilometer per second, and a very smooth Mg II BAL profile.

The combined properties of the Mg II , Mg I and Fe II lines seem to indicate that FBQS1044+3656 contains an outflow with BAL-like characteristics situated at a distance of a few hundred parsec from the nucleus, a scale very much larger than would have been attributed to the outflow on the basis of the Mg II lines only. At this time, FBQS1044+3656 is a unique object and we do not know how general these results are. In view of the importance of establishing the relation between the large scale outflow found here and the classical high ionization BAL phenomenon, the essential 
next step in the study of FBQS1044+3656 is to obtain a UV spectrum of the source to compare the absorption profiles of the low and high ionization species.

\section{ACKNOWLEDGMENTS}

Part of this work was performed under the auspices of the U.S. Department of Energy by University of California Lawrence Livermore National Laboratory under contract No. W-7405Eng-48. 


\section{REFERENCES}

Arav, N., \& Li, Z. Y. 1994, ApJ, 427, 700

Arav, N., Korista, K.T., de Kool, M., Junkkarinen, V.T. \& Begelman, M.C. 1999a, ApJ, 516, 27

Arav, N., Becker, R.H., Laurent-Muehleisen, S.A., Gregg, M.D., White, R.L. \& de Kool, M. 1999b, ApJ, 524,566.

Baldwin, J., Ferland, G.J., Martin, P.G., Corbin, M., Cota, S., Peterson, B.M., \& Slettebak, A., 1991, ApJ 374, 580

Barlow, T.A. 2000, AJ, in preparation

Barlow, T.A. \& Sargent, W.L.W. 1997, AJ, 113, 136

Begelman, M.C., de Kool, M. \& Sikora, M. 1991, ApJ, 382,416

Bergeson et al. 1996, ApJ, 464, 1044

Cardelli, J.A. \& Savage, B.D. 1995, ApJ, 452, 275

Collin-Souffrin, S., Joly, M., Pequignot, D. \& Dumont, S. 1986, A \& A, 166, 27

Ferland, G.J., 1996, Hazy, a Brief Introduction to Cloudy, University of Kentucky Department of Physics and Astronomy Internal Report.

Foltz, C.B. et al. 1986, ApJ, 307,504

Francis, P.J., Hewett, P.C., Foltz, C.B., Chafee, F.H., Weymann, R.J. \& Morris, S.L. 1991, ApJ, 373,465

Fuhr, R.J., Martin,G.A. \& Wiese,W.L. 1988, J. Phys. Chem. Ref. Data, 17, Suppl. 4, 108

Ganguly, R., Eracleous, M., Charlton, J.C. \& Churchill, C.W. 1999, ApJ, 117, 2594

Giridhar, S. \& Ferro, A.F. 1995, Rev. Mex. Astron. Astrofis., 31, 23

Hamann, F., Korista, T. K., \& Morris, S. L. 1993 ApJ, 415, 541

Hamann, F., Barlow, T.A., Beaver, E.A., Burbidge, E.M., Cohen, R.D., Junkkarinen, V.T. \& Lyons., R. 1995, ApJ, 443, 606

Hamann, F., Barlow, T.A., \& Junkkarinen, V.T. 1997, ApJ, 478, 87

Hamann, F.W., Netzer, H. \& Shields, J.C. 2000, ApJ, 536, 101

Laor, A., Fiore, F., Elvis,M., Wilkes, B.J. \& McDowell, J.C. 1997, ApJ, 477, 93

Mathews,W.G. \& Ferland, G. 1987, ApJ, 323, 456

Murray, N., Chiang, J., Grossman, S.A., \& Voit, G.M. 1995, ApJ, 451, 498

Nahar, S.N. 1995, A \& A 293, 967

Osterbrock, D. E., Tran, H.D., \& Veilleux, S., 1992, ApJ 389, 305

Petitjean, P. \& Srianand, R. 1999, A \& A, 345, 73

Raassen, A.J.J. \& Uylings, P.H.M. 1998, A \& A, 340, 300

Srianand,R. \& Petitjean, P. 2000, A \& A, 357, 414 
Turnshek, D. A. 1988, in Space Telescope Sci. Inst. Symp. 2, QSO Absorption Lines: Probing the Universe, ed. S. C. Blades, D. A. Turnshek, \& C. A. Norman (Cambridge: Cambridge Univ. Press), 17

Verner, E.M., Verner, D.A., Korista, K.T., Ferguson, J.W., Hamann, F., \& Ferland, G.J. 1999, ApJS, 120, 101

Vogt et al. 1994

Voit, G.M., Weymann, R.J. \& Korista, K.T. 1993, ApJ, 413, 95

Wampler, E.J., Chugai, N.N. \& Petitjean, P. 1995, ApJ, 443, 586 (WCP)

Weymann, R.J., Williams, R.E., Peterson, B.M. \& Turnshek, D.A. 1979, ApJ, 234, 33

Weymann, R. J., Turnshek, D. A., \& Christiansen, W. A. 1985, in Astrophysics of Active Galaxies and Quasi-stellar Objects, ed. J. Miller (Oxford: Oxford Univ. Press) 333 (WTC)

Weymann, R. J., Morris, S. L., Foltz, C.B. \& Hewett, P.C. 1991, ApJ, 373, 23

Weymann R.J. 1997, in ASP Conf. Ser. 128, Masss Ejection from AGN, ed. N. Arav, I. Shlosman, \& R.J. Weymann (San Francisco:ASP),3

White, R.L. et al. 2000, ApJS, 126, 133

Wills, B.J., Netzer, H, \& Wills, D. 1985, ApJ, 288, 94

Zhang, H.L. \& Pradhan, A.K. 1995, A \& A, 293, 953 H. Nussbaumer \& P. J. Storey, 1980, A \& A , 89, 308 Quinet P., Le Dourneuf M. \& Zeippen C.J., 1996, A \& A Suppl. 120, 361 
Fig. 1. - A smoothed plot of the spectrum of FBQS1044+3656, giving an overview of the regions analyzed in this work.

Fig. 2.- The absorption lines of $\mathrm{Mg} \mathrm{I}$ and $\mathrm{Mg}$ II as a function of velocity. The spectrum on the Mg I velocity scale has been offset by 0.3 for clarity. The Mg II velocity scale is based on the red component of the Mg II doublet. The line complexes discussed in the text are labelled.

Fig. 3. - The spectrum of FBQS1044+3656 in the region of the Fe II lines. The thin line represents the model fit based on the template fitting method and the parameters in Table 2 assuming the background continuum source is completely covered by the outflow. The model shown as the thick line is based on the same model parameters, but assumes that the covering factor as a function of velocity is given by the absorption profile of the strongest Fe II lines, as described in the text. The expected positions of the Fe II lines caused by the gas responsible for absorption components A,B,C and D of the Mg I profile (defined in Figure 2) are indicated above the spectrum. Lines of Mn II and the expected line positions for the strongest Fe I lines are also indicated.

Fig. 4. - The covering factor derived from a comparison of Fe II lines $\lambda 2600$ and $\lambda 2586$. The crosses represent the unobscured continuum flux $(1-\mathrm{C})$. The thin and thick lines are the profiles of the two lines used to obtain the solution for the covering factor. Contamination by other lines, noise and continuum errors allow no physical solution for the covering factor at some velocities. The positions at which this happens are indicated by an asterisk above the spectrum.

Fig. 5.- Comparison of the solutions for the unobscured flux in cluster 2 for 5 different pairs of lines from the Fe II ground state. Points with no physical solution have been omitted.

Fig. 6.- The structure of an irradiated slab with solar abundances as a function of ionization parameter. The variables are plotted as a function of position in the slab, normalized to the total thickness of the slab $D_{\max }$. The key next to the line type definition in panel (a) refers to the models in Table 3. Panel (a) shows the electron temperature, panel (b) the electron density and panels (c) and $(\mathrm{d})$ the ionization fraction of Fe I and Mg I respectively.

Fig. 7.- This figure illustrates how the ionization models in Figure 6 change if the spectrum of the incident radiation is a simple power law with slope -1.4 rather than the Matthews \& Ferland (1987) AGN spectrum.

Fig. 8.- Photoionized slab models assuming the gas contains dust. For the models with dust (Table 3) the elemental abundances in the gas phase are depleted as observed in the Orion nebula, except model 15 which has a lower $\mathrm{Mg}$ depletion. Since the higher $\mathrm{Mg}$ abundance has negligible influence on the slab structure it is indistinguishable from model 13 in these plots. Model 15 fulfills all observational constraints including those on the intrinsic extinction. 
Table 1. Fe II Lines And oscillator Strengths

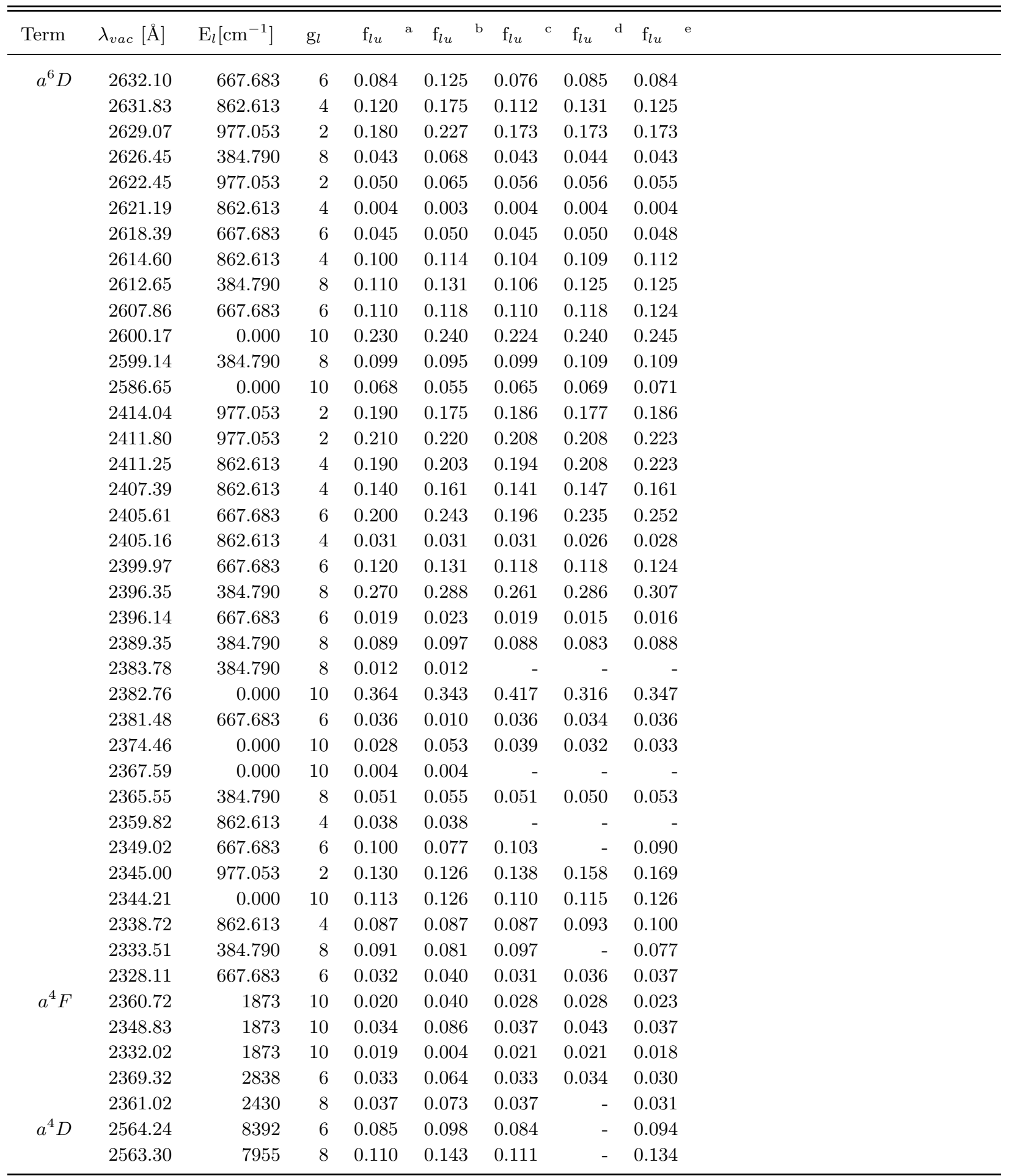

${ }^{a}$ NIST database, mostly Fuhr, Martin \& Wiese 1988, J. Phys. Chem. Ref. Data, 17, Suppl. 4, 108

${ }^{\mathrm{b}}$ Iron Project, Nahar 1995, A \& A 293, 967

${ }^{\mathrm{c}}$ Compilation of experimental data, Giridhar \& Ferro 1995, Rev. Mex. Astron. Astrofis., 31, 23

${ }^{\mathrm{d}}$ Experimental, Bergeson et al. 1996, ApJ, 464, 1044

${ }^{\mathrm{e}}$ Raassen \& Uylings 1998, A \& A, 340, 300 
Table 2. Relative population of Fe il energy LEVELS

\begin{tabular}{cccc}
\hline \hline Term & Energy $\left[\mathrm{cm}^{-1}\right]$ & $\zeta(1)$ & $\zeta(2)$ \\
\hline$a^{6} D$ & 0. & 1.00 & 1.00 \\
& 384. & $0.04(0.02)$ & $0.12(0.03)$ \\
& 667. & $0.04(0.02)$ & $0.10(0.03)$ \\
& 862. & $0.04(0.03)$ & $0.10(0.03)$ \\
& 977. & $<0.1$ & $0.10(0.03)$ \\
$a^{4} F$ & 1873. & $<0.3$ & $0.50(0.1)$ \\
& 2430. & $<0.6$ & $<0.2$ \\
& 2838. & $<0.6$ & $<0.2$ \\
$a^{4} D$ & 7955. & $<0.1$ & $<0.03$ \\
& 8392. & $<0.2$ & $<0.1$ \\
\hline
\end{tabular}

Note. - The relative population per unit statistical weight of the low-lying energy levels of Fe II that were derived from the relative strengths of the absorption lines. $\zeta$ would be equal to 1 if the level population were in LTE at a temperature higher than the level energy. $\zeta(1)$ applies to the low velocity line cluster, $\zeta(2)$ to the high velocity cluster. Note that the errors only reflect the fitting uncertainty, and do not include systematic errors like uncertainties in the continuum. 
Table 3. IONIZATION MODELS

\begin{tabular}{|c|c|c|c|c|c|c|c|c|c|c|}
\hline Model \# & $\log U$ & $\log n_{H}$ & spectrum $^{a}$ & abundance & ${ }^{b}$ dust ${ }^{b}$ & b $\log N_{H}{ }^{\mathrm{c}}$ & ${ }^{\mathrm{c}} \log N_{M g I}{ }^{\mathrm{d}}$ & $\log N_{F e I}{ }^{\mathrm{e}}$ & $A_{2500}$ & f Distance (kpc) \\
\hline 1 & -1.0 & 3.90 & $\mathrm{AGN}$ & solar & no & 22.00 & 12.45 & 10.44 & - & 0.24 \\
\hline 2 & -2.0 & 4.00 & AGN & solar & no & 21.01 & 12.89 & 11.23 & - & 0.67 \\
\hline 3 & -3.0 & 4.10 & AGN & solar & no & 20.25 & 12.75 & 11.66 & - & 1.9 \\
\hline 4 & -4.0 & 4.20 & AGN & solar & no & $?$ & $>12.47$ & $>12.09$ & - & 5.3 \\
\hline 5 & -5.0 & 4.50 & $\mathrm{AGN}$ & solar & no & $?$ & $>12.82$ & $>12.56$ & - & 19 \\
\hline 6 & -6.0 & 4.90 & AGN & solar & no & $?$ & $>12.82$ & $>13.20$ & - & 94 \\
\hline 7 & -2.0 & 4.00 & AGN & $3 \times$ solar & no & 20.97 & 13.35 & 11.48 & - & 0.67 \\
\hline 8 & -2.0 & 4.00 & power law & solar & no & 21.03 & 12.74 & 11.21 & - & 0.53 \\
\hline 9 & -4.0 & 4.20 & power law & solar & no & 20.00 & 12.34 & 12.00 & - & 4.2 \\
\hline 10 & -6.0 & 4.90 & power law & solar & no & $?$ & $>12.79$ & $>13.06$ & - & 74 \\
\hline 11 & -1.0 & 4.60 & AGN & Orion & yes & 21.70 & 12.61 & 11.88 & 4.2 & 0.10 \\
\hline 12 & -2.0 & 5.00 & AGN & Orion & yes & 21.26 & 12.49 & 11.67 & 1.5 & 0.21 \\
\hline 13 & -3.0 & 5.00 & AGN & Orion & yes & 21.06 & 12.86 & 12.30 & 0.96 & 0.66 \\
\hline 14 & -4.0 & 5.00 & AGN & Orion & yes & $?$ & $>12.65$ & $>12.42$ & $?$ & 2.1 \\
\hline 15 & -3.0 & 5.00 & AGN & Orion $\mathrm{Mg} \times 3$ & yes & 21.06 & 13.34 & 12.30 & 0.96 & 0.66 \\
\hline
\end{tabular}

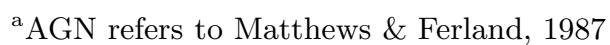

${ }^{\mathrm{b}}$ Abundances and dust properties based on values for the Orion nebula, Baldwin et al. 1991,Osterbrock et al. 1992

${ }^{\mathrm{c}}$ Total hydrogen column density up to $\log N_{F e} I I=15.5$

${ }^{\mathrm{d}}$ Total $\mathrm{Mg}$ I column density up to $\log N_{F e}$ II $=15.5$

${ }^{\text {e }}$ Total Fe I column density up to $\log N_{F e} I I=15.5$

${ }^{\mathrm{f}}$ Expected dust extinction at $2500 \AA$ for the models with dust

${ }^{\mathrm{g}}$ Distance implied by density and ionization parameter assuming a QSO luminosity of $10^{46} \mathrm{ergs}^{-1}$ 


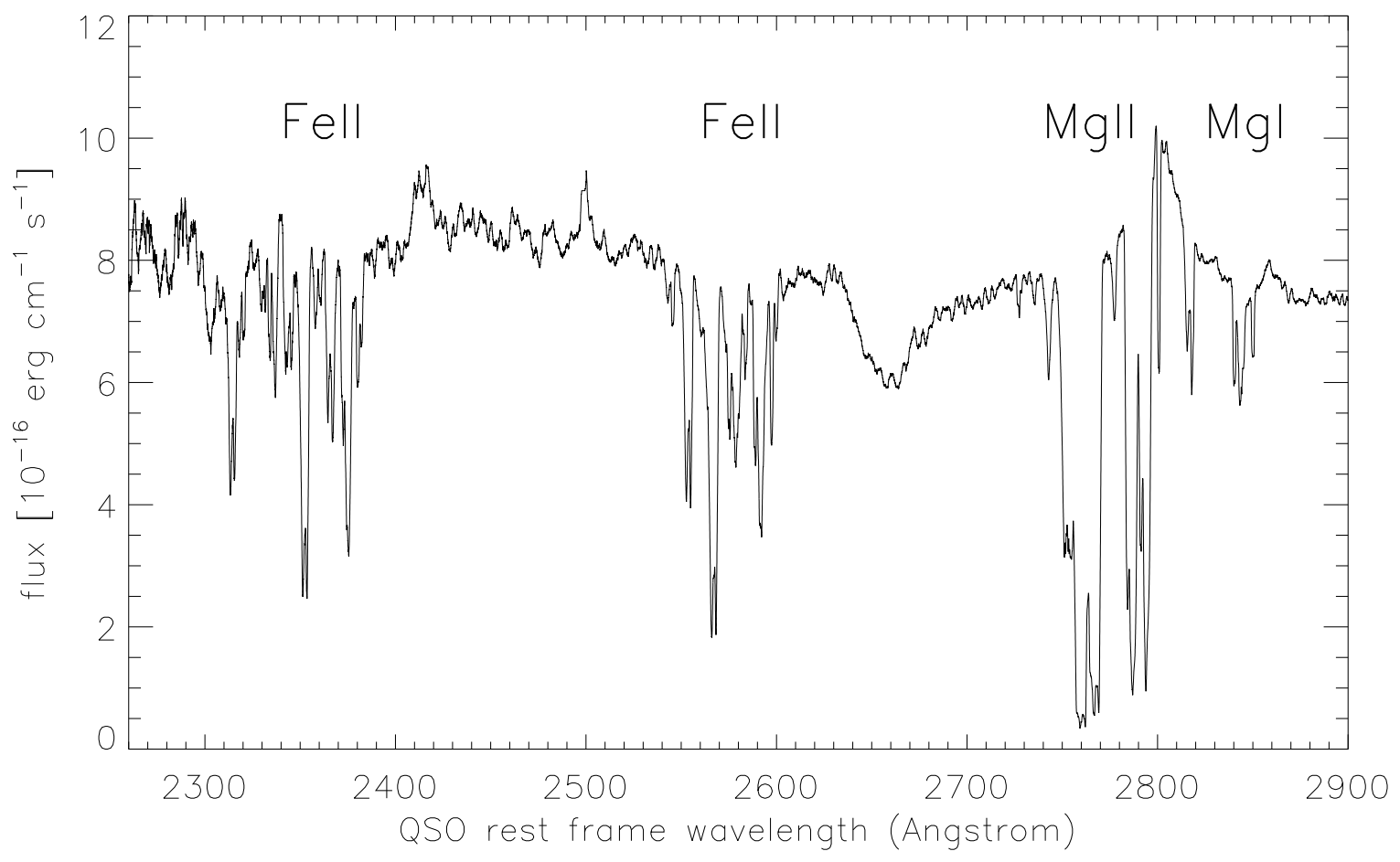



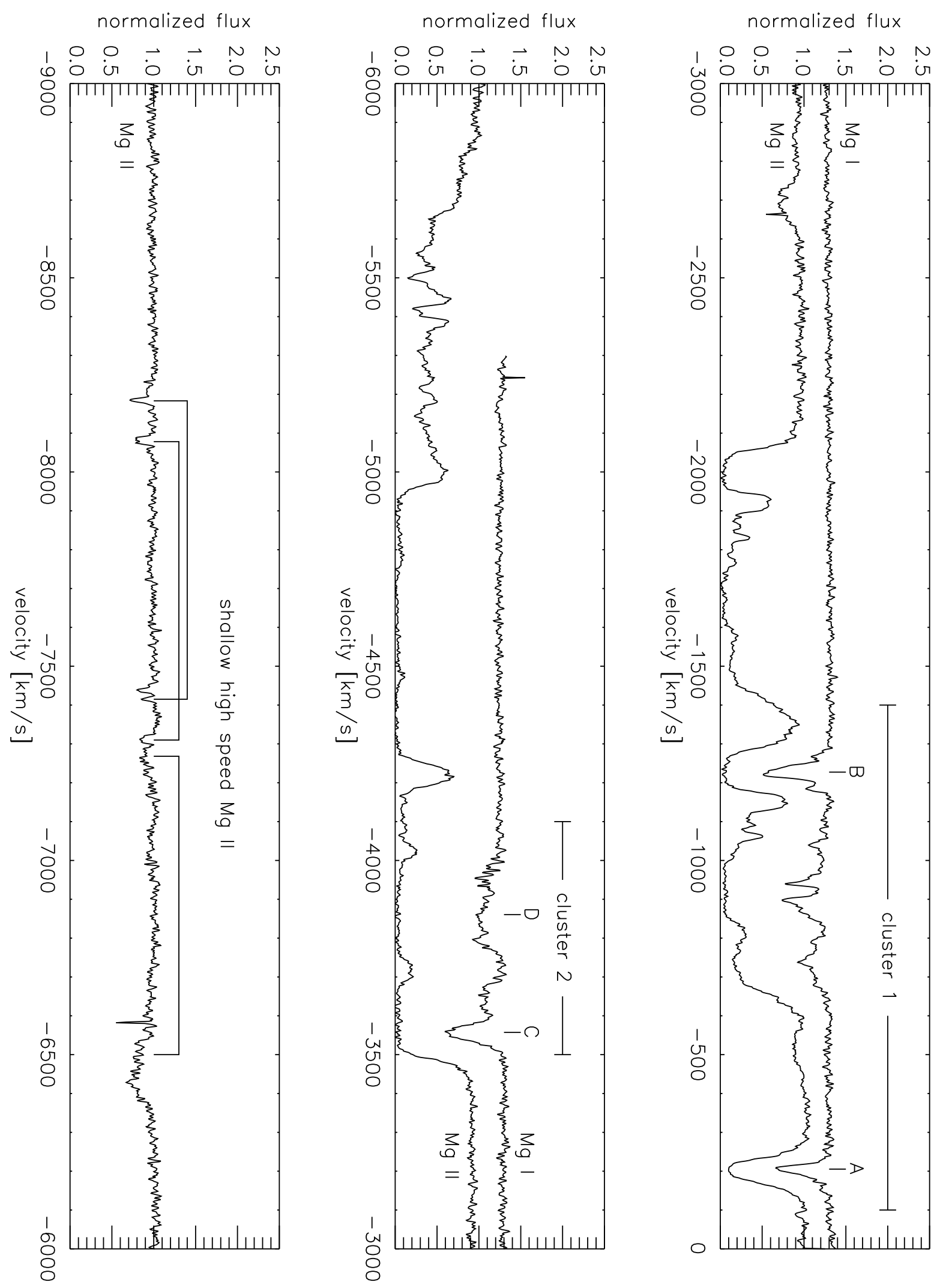

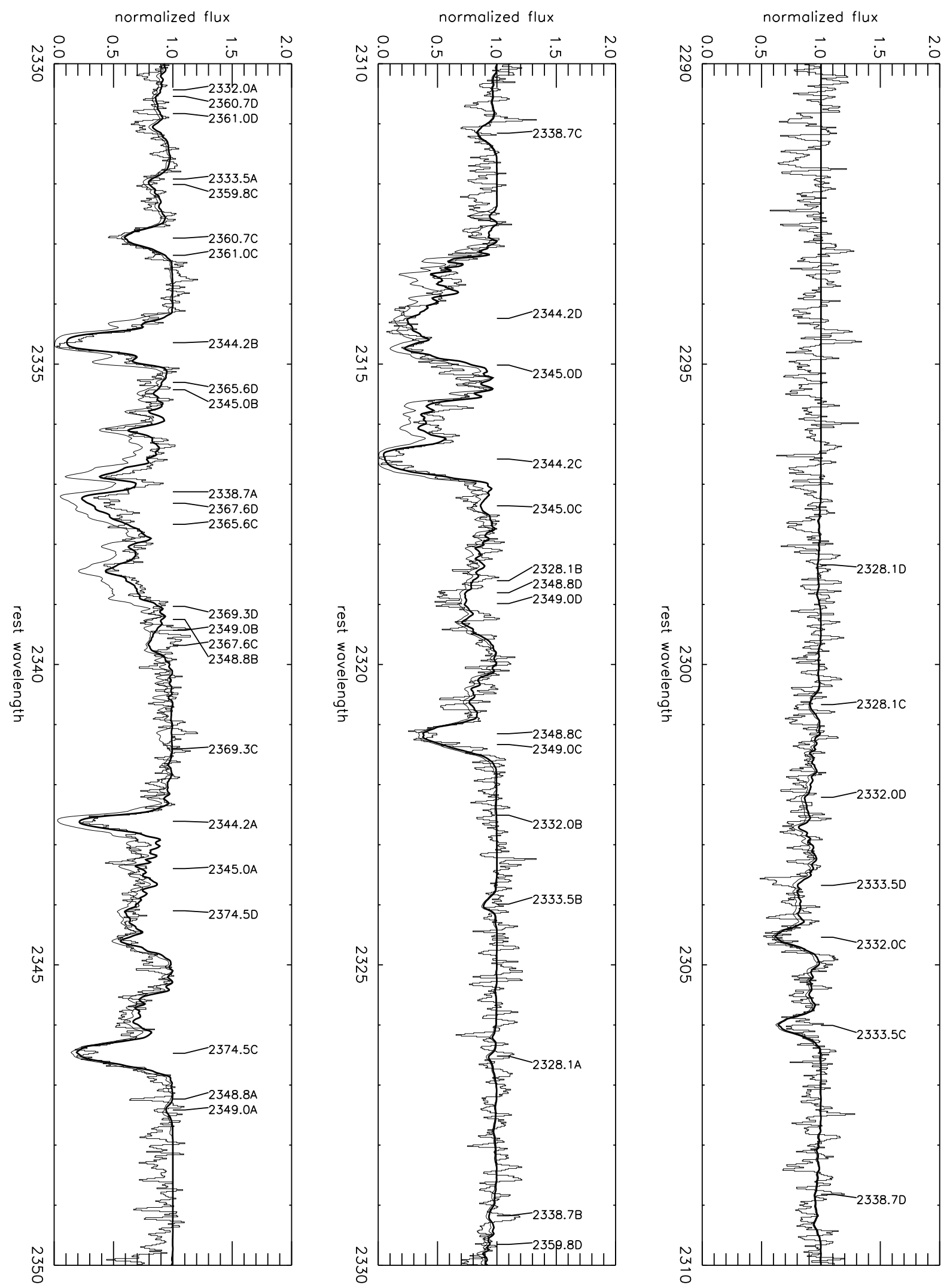

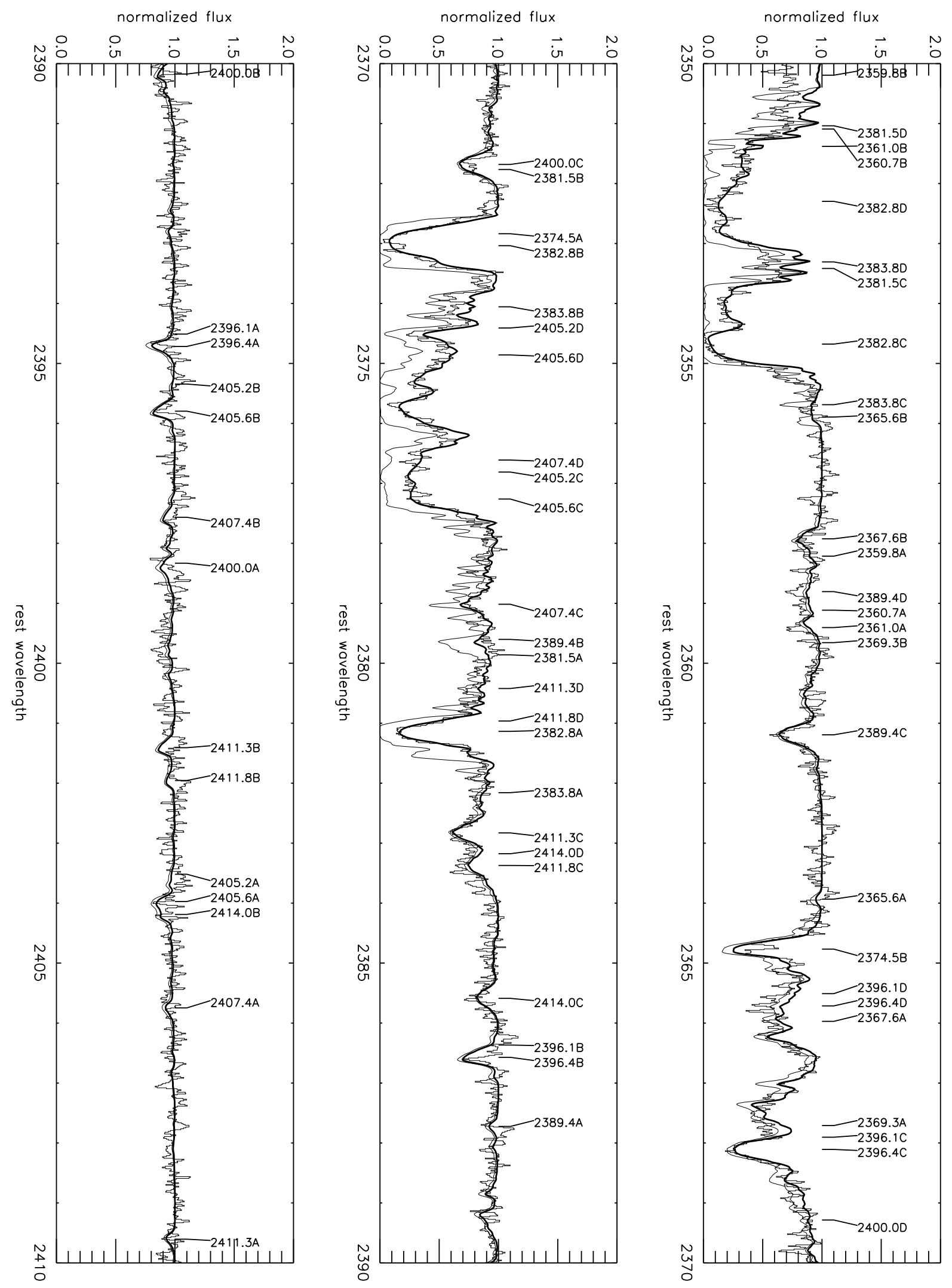

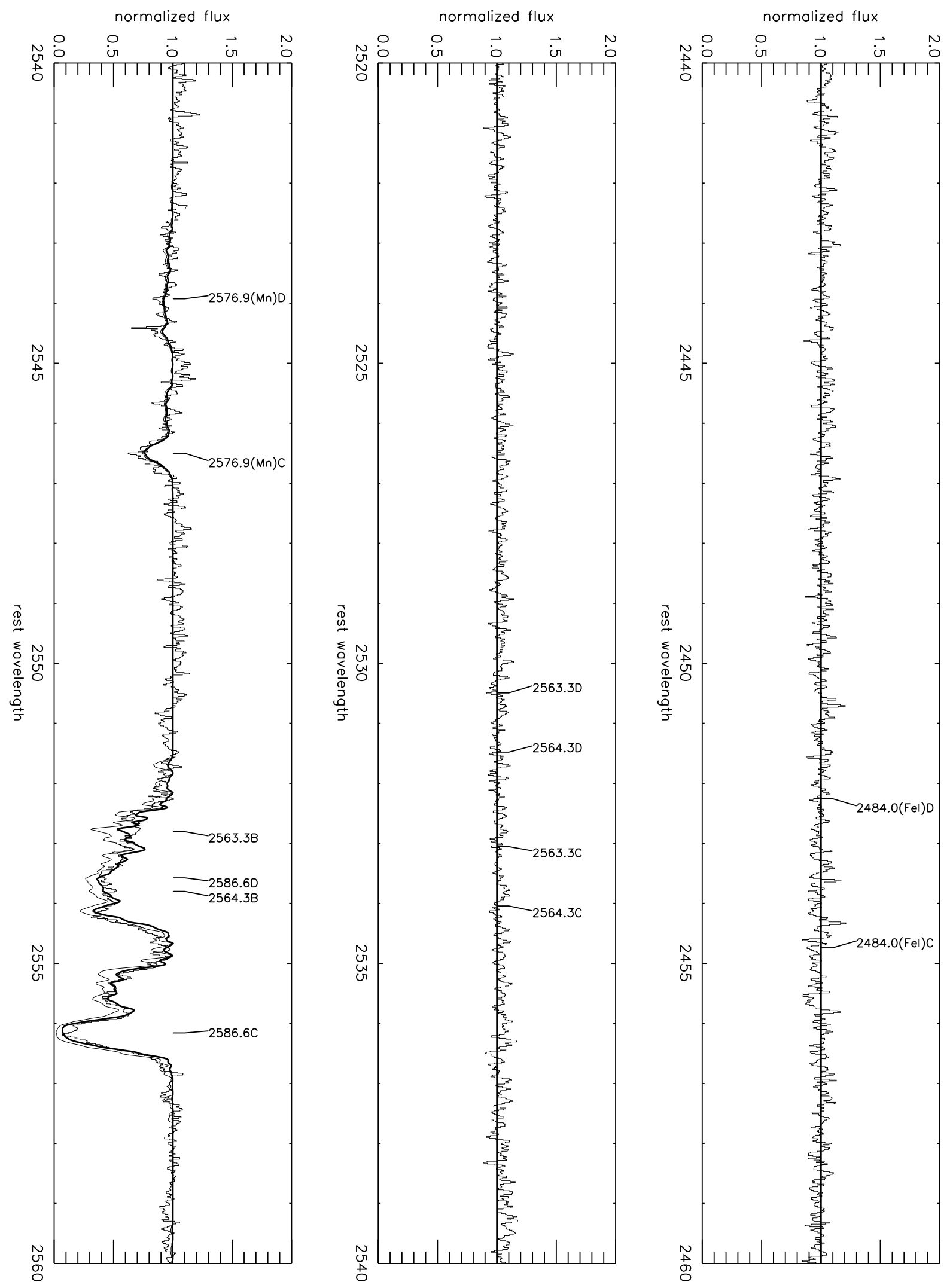

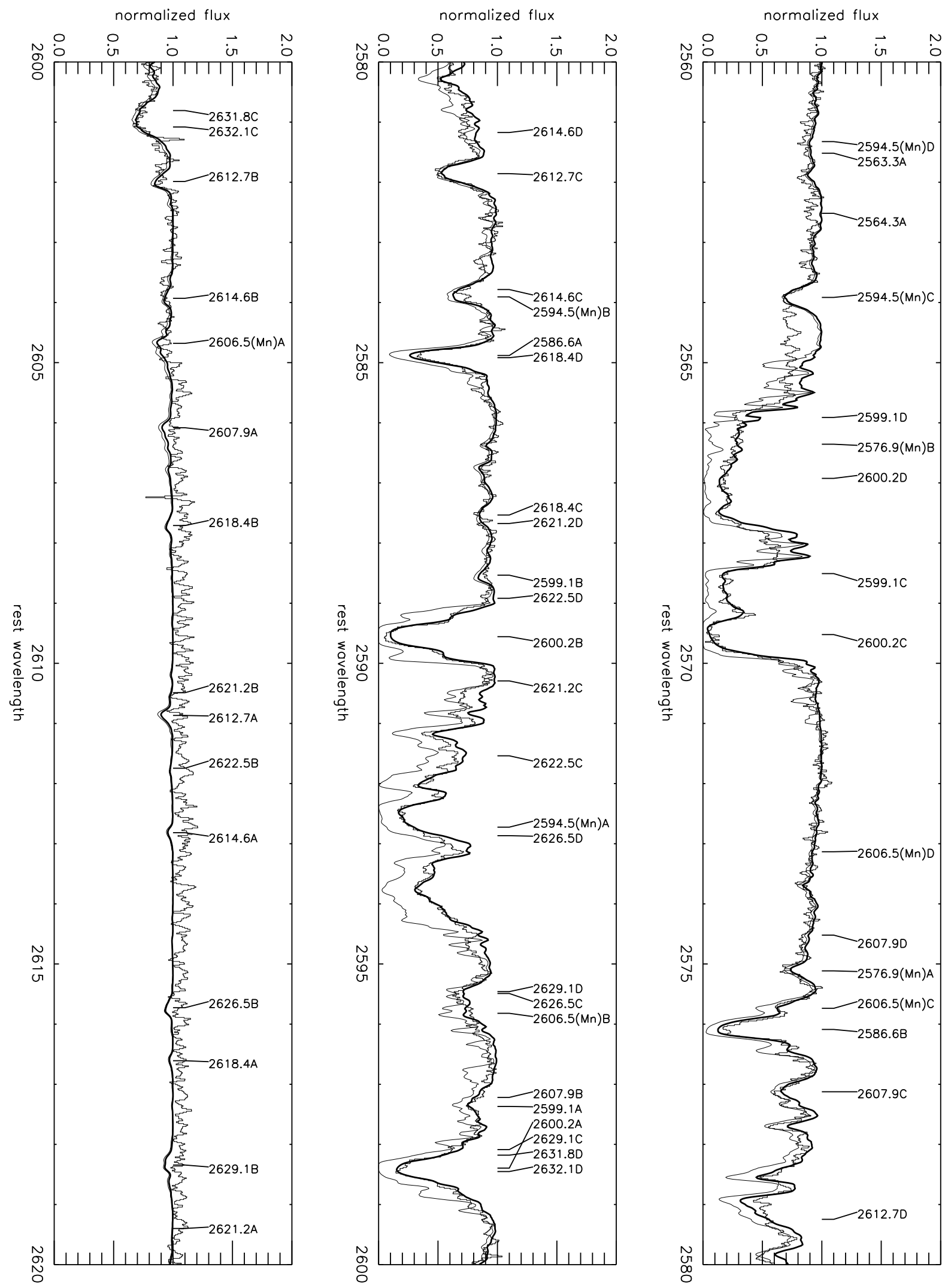

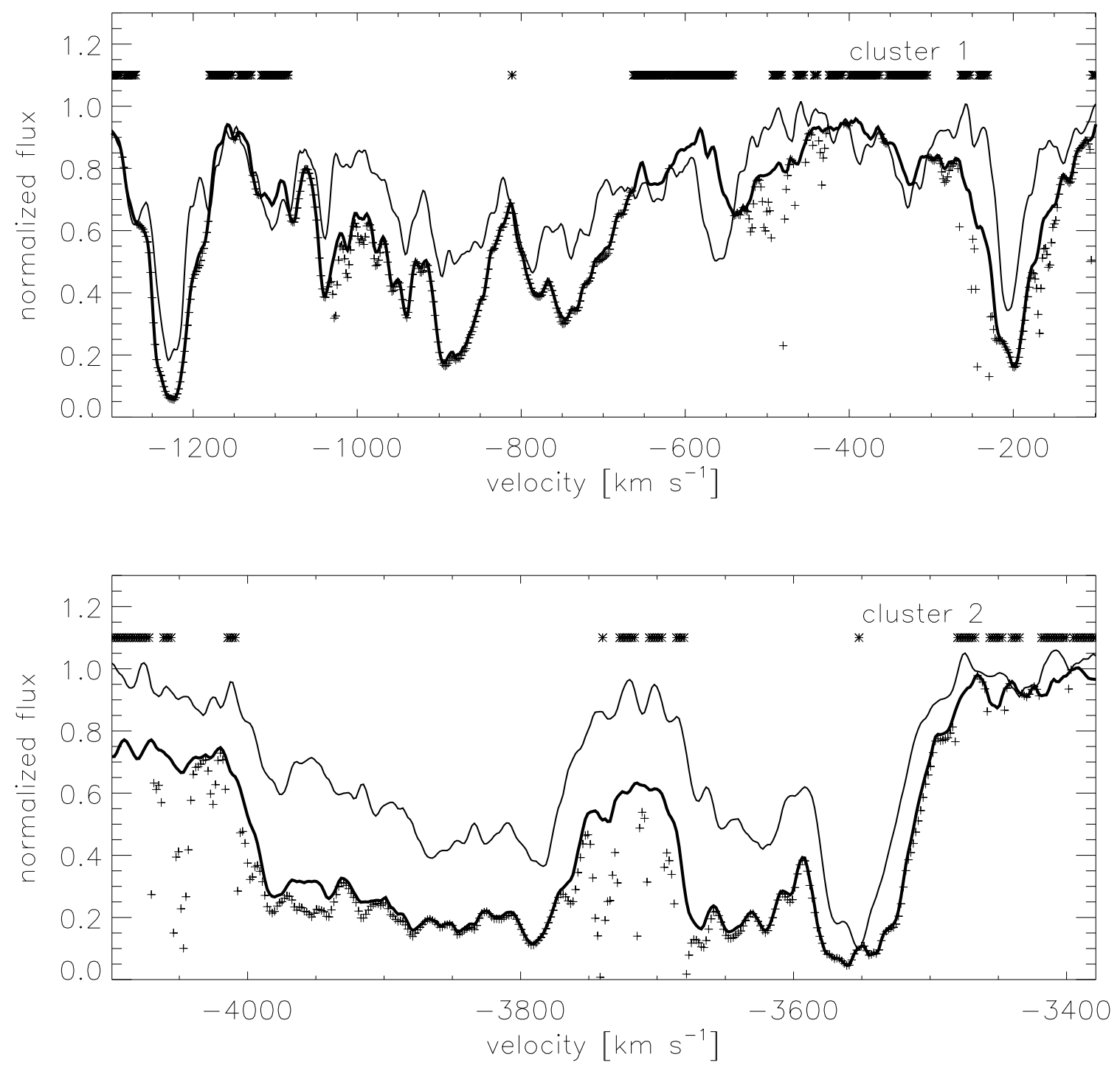


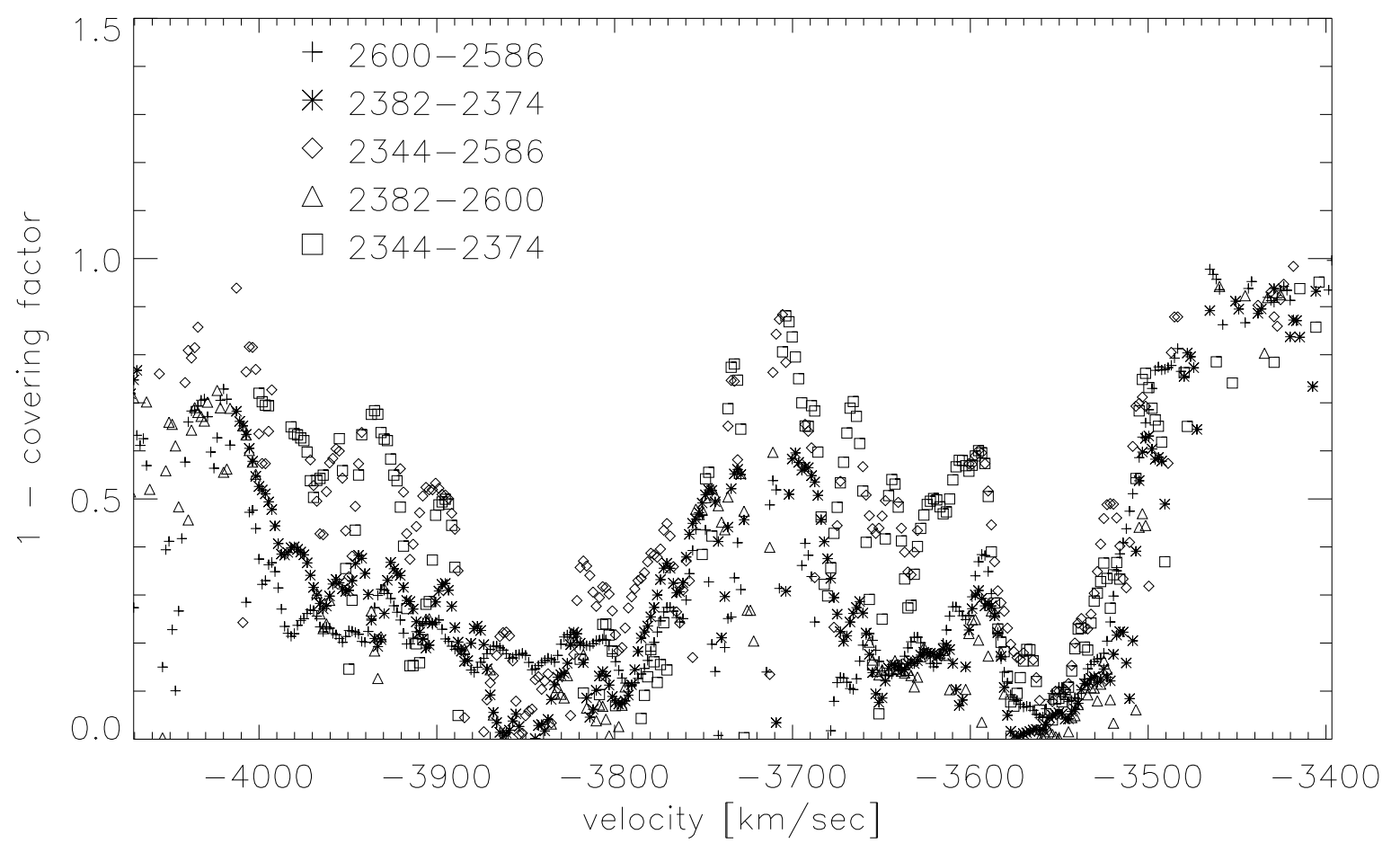



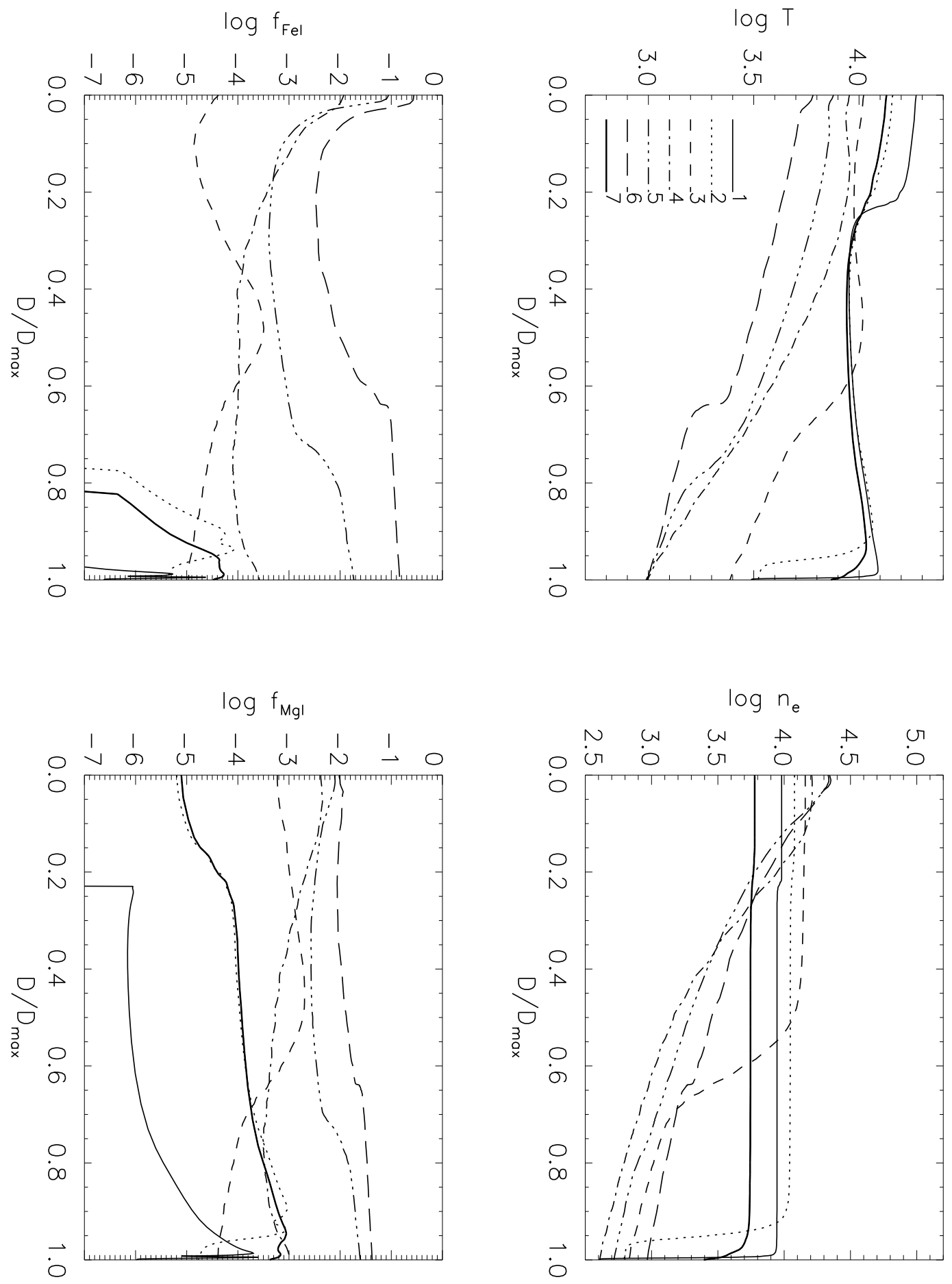

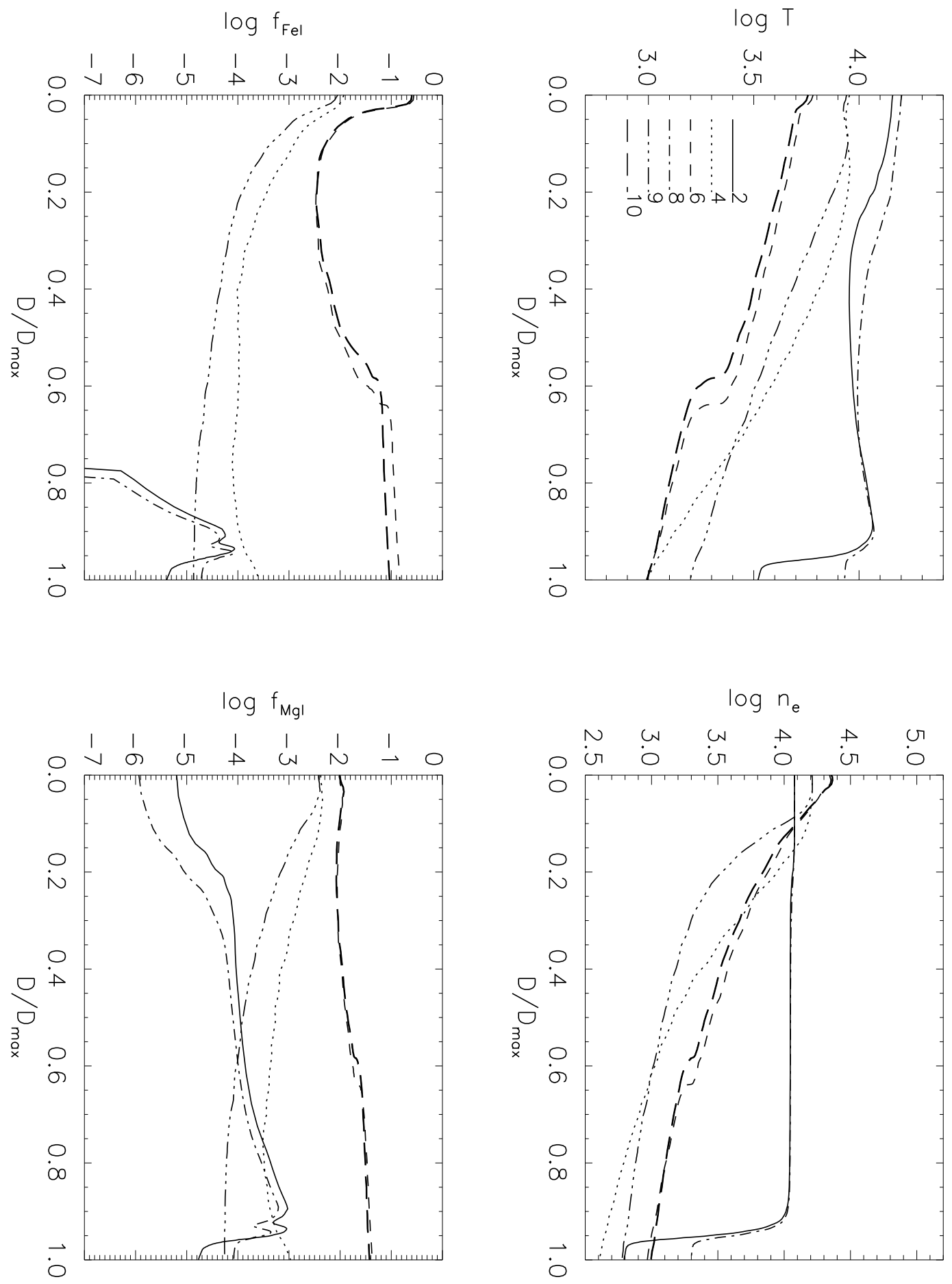

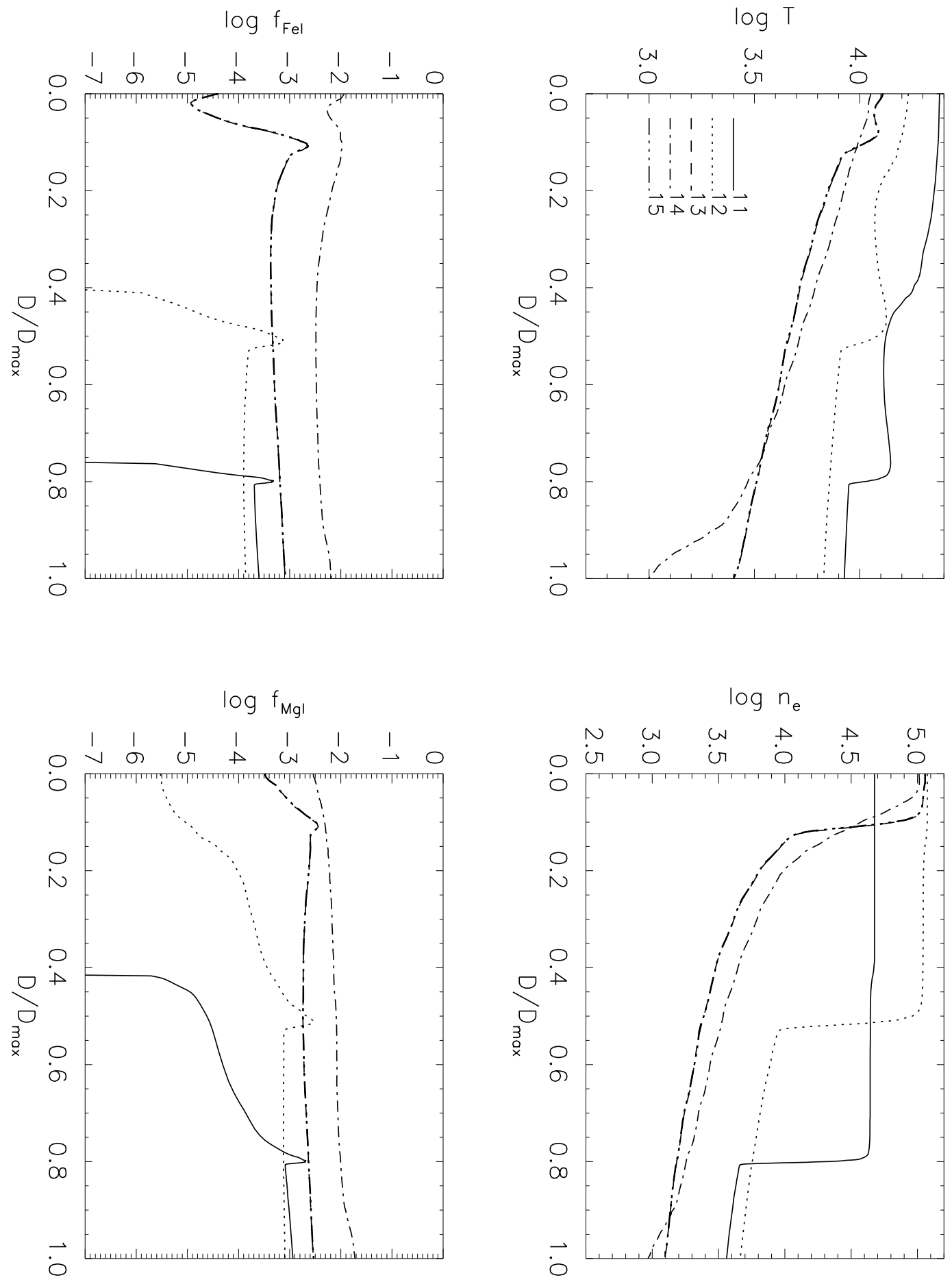